\title{
A finite strain, finite band method for modeling ductile fracture
}

\author{
A.E. Huespe ${ }^{\mathrm{a}, *}$, A. Needleman ${ }^{\mathrm{b}}$, J. Oliver ${ }^{\mathrm{c}}$, P.J. Sánchez ${ }^{\mathrm{a}, \mathrm{d}}$ \\ ${ }^{a}$ CIMEC-INTEC-UNL-CONICET, Güemes 3450, 3000 Santa Fe, Argentina \\ ${ }^{\mathrm{b}}$ Department of Materials Science and Engineering, College of Engineering and Center for Advanced Scientific Computing and Modeling, \\ University of North Texas, Denton, TX, United States \\ ${ }^{\mathrm{c}}$ ETSECCP, Technical University of Catalonia, Barcelona, Spain \\ ${ }^{\mathrm{d}}$ GIMNI-UTN-FRSF, Lavaisse 610, 3000 Santa Fe, Argentina
}

\section{A R T I C L E I N F O}

\section{Article history:}

Received 16 February 2011

Received in final revised form 17 May 2011

Available online 14 June 2011

\section{Keywords:}

Ductile fracture modeling

Finite element methods

Finite strain plasticity

Embedded weak discontinuities

Regularization

\begin{abstract}
A B S T R A C T
We present a finite deformation generalization of the finite thickness embedded discontinuity formulation presented in our previous paper [A.E. Huespe, A. Needleman, J. Oliver, P.J. Sánchez, A finite thickness band method for ductile fracture analysis, Int. J. Plasticity 25 (2009) 2349-2365]. In this framework the transition from a weak discontinuity to a strong discontinuity can occur using a single constitutive relation which is of importance in a range of applications, in particular ductile fracture, where localization typically precedes the creation of new free surface. An embedded weak discontinuity is introduced when the loss of ellipticity condition is met. The resulting localized deformation band is given a specified thickness which introduces a length scale thus providing a regularization of the post-localization response. The methodology is illustrated through several example problems emphasizing finite deformation effects including the development of a cup-cone failure in round bar tension.
\end{abstract}

(c) 2011 Elsevier Ltd. All rights reserved.

\section{Introduction}

Predicting the creation of new free surface necessarily involves introducing a length scale if only from dimensional considerations since, for example, the work of separation of two surfaces has the dimensions of $\mathrm{J} / \mathrm{m}^{2}$. For ductile fracture, there is another issue since the creation of new free surface is often preceded by the localization of deformation into a narrow band and a length scale is needed to set the band width.

A variety of ways to introduce a length scale into continuum formulations have been proposed. One way is by introducing higher order terms into the constitutive relation for plastic flow based on considerations related to the micromechanics of deformation, as for example in Fleck and Hutchinson (1997) and Gao et al. (1999). Approaches to introducing a length scale in ductile fracture modeling are presented in Mediavilla et al. (2006), Bargellini et al. (2009), Cazes et al. (2010), and Huespe et al. (2009).

In Huespe et al. (2009), we presented a finite element method with a finite thickness embedded weak discontinuity to analyze ductile fracture problems that was restricted to small geometry changes. An embedded weak discontinuity was introduced when the loss of ellipticity condition was met. A material length scale was introduced to give the resulting localized deformation band a specified thickness. Within the band, the deformation is specified to be homogeneous and is governed by the pre-localization constitutive relation. As a consequence, convergent calculations of the history of deformation through localization and the creation of new free surface can be carried out. It was also shown that in the limit of vanishing band thickness a cohesive surface formulation is approached with the important difference that for a finite band width the

\footnotetext{
* Corresponding author.

E-mail address: ahuespe@intec.unl.edu.ar (A.E. Huespe).
} 
separation relation can be hydrostatic stress dependent. In essence, the methodology allows for a unified framework for analyzing the transition from a weak discontinuity to a strong discontinuity.

Here, we extend the formulation in Huespe et al. (2009) to finite deformations. This is of importance in ductile fracture analyses since large strains generally occur prior to fracture, at least locally. As in our small deformation analyses, the calculations carried out are based on the rate independent constitutive relation for progressively cavitating solids introduced by Gurson (1975) and as modified by Tvergaard $(1981,1982)$ and Tvergaard and Needleman (1984). Recent comparisons of numerical predictions with experiments are presented in Gao et al. (2011) and Li et al. (2011). A number of recent studies, for example Benzerga and Leblond (2010) and Pardoen et al. (2010), have been carried out aimed at developing more accurate models of void nucleation, growth and coalescence. Other studies have been aimed at improving predictions for the dependence on stress state, particularly at low values of the stress triaxiality, Nashon and Hutchinson (2008) and Gao et al. (2011), but the basic framework remains.

It is, however, worth noting that the present general formulation can be applied to any rate independent constitutive relation that gives rise to localized deformation and, possibly, creation of new free surface.

\section{Governing equations}

\subsection{Field equations}

Consider a body occupying the domain $\Omega(t)$ at time $t$, which is subjected to a quasi-static loading condition. The body position at time $t=0:\left(\Omega_{0}=\Omega(0)\right)$ is adopted as the reference configuration. Given the deformation map: $\boldsymbol{x}=\varphi(\boldsymbol{X})$, $\Omega_{0} \rightarrow \Omega(t)$, where $\boldsymbol{x}$ denotes the position of a material point in the current configuration and $\boldsymbol{X}$ the position of that material point in the reference configuration, the principle of virtual work, with body forces neglected, is written as

$$
\int_{\Omega_{0}} J \sigma \boldsymbol{F}^{-T}: \nabla_{\boldsymbol{X}} \delta \boldsymbol{u} d \Omega_{0}-\int_{\Gamma_{\sigma}} \boldsymbol{t} \cdot \delta \boldsymbol{u} d \Gamma=0 ; \quad \forall \delta \boldsymbol{u} \in \mathcal{V}_{0},
$$

where $\nabla_{\mathbf{X}}$ is the (material) gradient operator, $\mathbb{F}$ is the gradient of $\varphi: \mathbb{F}=\nabla_{\mathbf{X}} \varphi, J$ is its Jacobian: $J=\operatorname{det}(\boldsymbol{F}), \sigma$ is the Cauchy stress
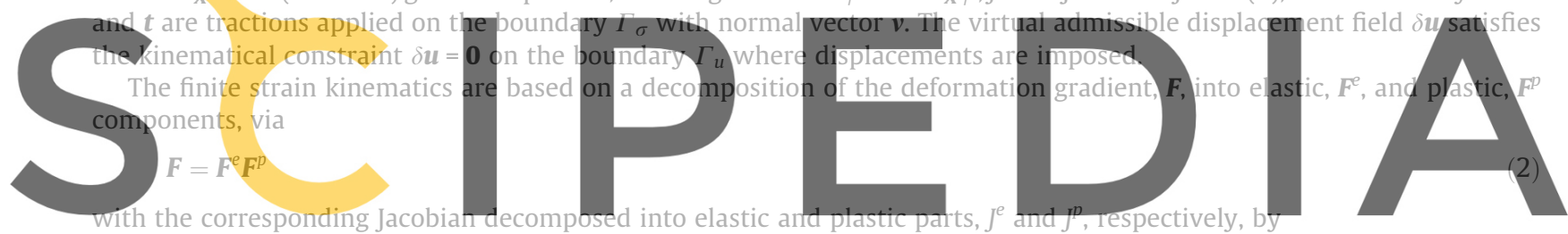

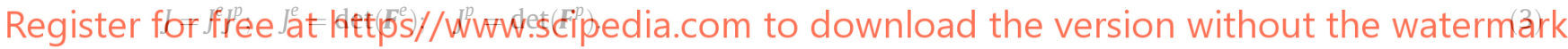

The velocity gradient, $\boldsymbol{l}$, is written as the sum of elastic and plastic parts, denoted by $\boldsymbol{l}^{e}$ and $\boldsymbol{l}^{p}$.

$$
\begin{aligned}
& \boldsymbol{l}=\dot{\boldsymbol{F}} \boldsymbol{F}^{-1}=\dot{\boldsymbol{F}}^{e} \boldsymbol{F}^{e-1}+\boldsymbol{F}^{e}(\underbrace{\dot{\boldsymbol{F}}^{p} \boldsymbol{F}^{p-1}}_{\mathbf{L}^{p}}) \boldsymbol{F}^{e-1}=\boldsymbol{l}^{e}+\boldsymbol{l}^{p}, \\
& \boldsymbol{l}^{e}=\dot{\boldsymbol{F}}^{e} \boldsymbol{F}^{e-1} ; \quad \boldsymbol{l}^{p}=\boldsymbol{F}^{e} \boldsymbol{L}^{p} \boldsymbol{F}^{e-1} .
\end{aligned}
$$

Also, the strain rate, $\boldsymbol{d}$, is decomposed into elastic and plastic components, denoted as $\boldsymbol{d}^{e}$ and $\boldsymbol{d}^{p}$ which are defined by

$$
\boldsymbol{d}=\boldsymbol{d}^{e}+\boldsymbol{d}^{p} ; \quad \boldsymbol{d}^{e}=\frac{1}{2}\left[\boldsymbol{l}^{e}+\left(\boldsymbol{l}^{e}\right)^{T}\right] ; \quad \boldsymbol{d}^{p}=\frac{1}{2}\left[\boldsymbol{l}^{p}+\left(\boldsymbol{l}^{p}\right)^{T}\right] .
$$

The constitutive relation will specify expressions for $\boldsymbol{d}^{e}$ and $\boldsymbol{d}^{p}$. The spin, the antisymmetric part of $\boldsymbol{l}$, is not constitutively specified.

\subsection{Constitutive relation}

The constitutive relation used is the micromechanically based material model for progressively cavitating plastic solids introduced by Gurson (1975) and subsequently modified, Tvergaard (1981, 1982) and Tvergaard and Needleman (1984). Here, this constitutive relation is briefly presented in the notation we use. Background and a more complete description of the constitutive framework are given in Tvergaard (1990).

Although elastic strains almost always remain small in the quasi-static ductile fracture problems of interest, possible finite elastic strains are accounted for in the formulation here. The elastic response of material is taken to be isotropic with the Cauchy stress, $\boldsymbol{\sigma}$, expressed as a function of the elastic left stretch tensor $\boldsymbol{V}^{e}, \boldsymbol{V}^{e}=\sqrt{\boldsymbol{F}^{e} \boldsymbol{F}^{e^{T}}}$, through

$$
\boldsymbol{\sigma}=\frac{\tau}{J^{e}}=\frac{1}{J^{e}} \frac{E}{(1+v)}\left[\ln \boldsymbol{V}^{e}+\frac{v}{(1-2 v)} \operatorname{tr}\left(\ln \boldsymbol{V}^{e}\right) 1\right],
$$


where $\tau$ is the second Piola-Kirchhoff stress tensor, $E$ is Young's modulus and $v$ is Poisson's ratio. In rate form, this gives

$$
\mathcal{L}^{e}(\tau)=\boldsymbol{c}^{e}: \boldsymbol{d}^{e}
$$

relating the convected rate of the (Kirchhoff) stress tensor, $\mathcal{L}^{e}(\boldsymbol{\tau})=\dot{\boldsymbol{\tau}}-\boldsymbol{l}^{e} \boldsymbol{\tau}-\boldsymbol{\tau}\left(\boldsymbol{l}^{e}\right)^{T}$, to the elastic strain rate $\boldsymbol{d}^{e}$ through the tensor of the elastic moduli $\boldsymbol{c}^{e}$.

With the spectral decomposition of $\boldsymbol{V}^{e}$ according to: $\boldsymbol{V}^{e}=\lambda_{I}\left(\boldsymbol{r}_{I} \otimes \boldsymbol{r}_{I}\right)$, being $\lambda_{I}$ and $\boldsymbol{r}_{I}$ its $I$-th principal value and principal stretch direction respectively; the tensor $\boldsymbol{c}^{e}$, consistent with the elastic relation Eq. (7), is expressed in the direction of principal stretches, which is a convenient form for numerical implementation, see e.g. Simo (1998):

$$
\boldsymbol{c}^{e}=\left[\overline{\boldsymbol{c}}_{I J}\left(\boldsymbol{r}_{I} \otimes \boldsymbol{r}_{I} \otimes \boldsymbol{r}_{J} \otimes \boldsymbol{r}_{J}\right)+\mathbb{b} ; \quad I, J=1,2 \quad\left(\text { in } \mathbb{R}^{2}\right)\right.
$$

for the two dimensional problems considered here (the generalization to three dimensions is straightforward).

The term $\bar{c}$ is given by:

$$
\overline{\boldsymbol{c}}=\frac{E}{(1+v)}\left[\boldsymbol{I}+\frac{v}{(1-2 v)} \mathbb{1} \otimes \mathbb{1}\right]
$$

with $\mathbb{1}=\{1,1\}$ (in $\mathbb{R}^{2}$ ), $\boldsymbol{I}$ is the identity tensor, and the tensor $\mathbb{t}$ is given by

$$
\mathbb{h}_{\mathfrak{L}}=\left\{\begin{array}{l}
\mathbb{h}_{I I I I}=-2 \tau_{I}, \\
\mathfrak{l}_{I J I J}=\frac{\tau_{I} \lambda_{j}-\tau_{J} \lambda_{I}}{\lambda_{1}-\lambda_{J}} ;
\end{array} \text { for } I \neq J,\right.
$$

where $\tau_{J}$ is the value of the $J$-th principal Kirchhoff stress (in $\mathbb{R}^{2}$ ). The change in the principal directions of stress with deformation gives rise to the th term, see for example Hill (1978).

In the context of the multiplicative decomposition, Eq. (2), consistency between the definition of the strain rates in Eqs. (6) and (8) is obtained by consideration of an appropriate intermediate configuration, as discussed e.g. in Lubarda (2002).

The plastic strain rate $\boldsymbol{d}^{p}$ is given by the plastic flow rule

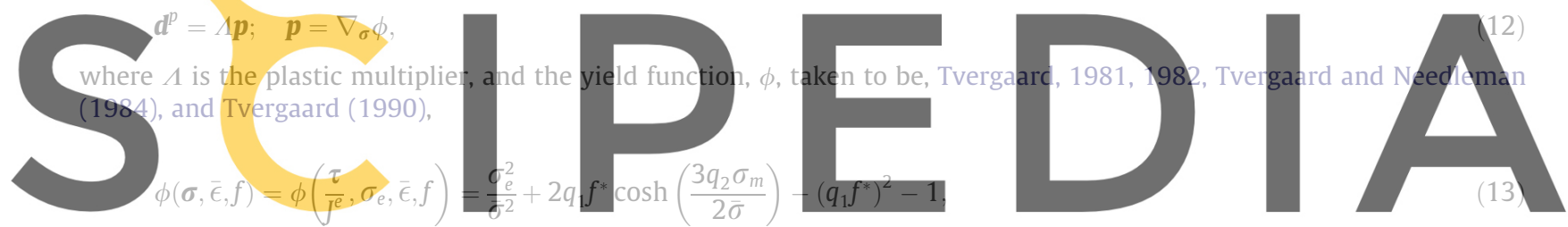

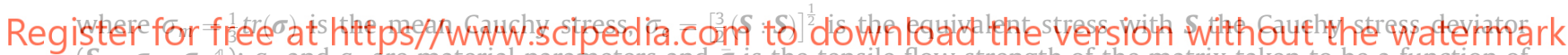
$\left(S=\sigma-\sigma_{m} 1\right) ; q_{1}$ and $q_{2}$ are material parameters and $\sigma$ is the tensile flow strength of the matrix taken to be a function of the matrix equivalent plastic strain $\bar{\epsilon}$ through the hardening relation

$$
\bar{\sigma}=\bar{\sigma}(\bar{\epsilon})=\sigma_{y}\left[1+\frac{\bar{\epsilon}}{\epsilon_{0}}\right]^{N}
$$

with $\sigma_{y}$ and $N$ being the initial matrix yield strength and the hardening exponent, respectively, and $\epsilon_{0}$ is a parameter normalizing the matrix equivalent plastic strain.

The function $f^{*}$, which was introduced in Tvergaard and Needleman (1984) to account for an increased void growth rate near coalescence is specified as

$$
f^{*}= \begin{cases}f & \text { for } f \leqslant f_{c}, \\ f_{c}+\frac{\frac{1}{q_{1}}-f_{c}}{f_{f}-f_{c}}\left(f-f_{c}\right) & \text { for } f_{c}<f \leqslant f_{f},\end{cases}
$$

where $f_{c}$ is the void volume fraction at which coalescence starts and $f^{*}\left(f_{f}\right)=1 / q_{1}$ is the void fraction at which the material loses all stress carrying capacity, i.e. $\phi=0$, such that $\sigma_{m}=0$ and $\sigma_{e}=0$.

The evolution of the void volume fraction is governed by

$$
\dot{f}=(1-f) \operatorname{tr}\left(\boldsymbol{d}^{p}\right)+\mathcal{A}_{m}(\bar{\epsilon}) \dot{\bar{\epsilon}}
$$

with the first term accounting for the change in void volume fraction due to the growth of existing voids and the second term for the change in void volume fraction due to the nucleation of new voids via strain controlled nucleation with $\mathcal{A}_{m}(\bar{\epsilon})$ given by, Chu and Needleman (1980),

$$
\mathcal{A}_{m}(\bar{\epsilon})=\frac{f_{N}}{s_{N} \sqrt{2 \pi}} \exp \left[-\frac{1}{2}\left(\frac{\bar{\epsilon}-\bar{\epsilon}_{N}}{s_{N}}\right)^{2}\right],
$$


where $f_{N}, s_{N}$ and $\bar{\epsilon}_{N}$ are material parameters.

The matrix plastic strain rate is given by

$$
\dot{\bar{\epsilon}}=\frac{\boldsymbol{\sigma}: \boldsymbol{d}^{p}}{(1-f) \bar{\sigma}} .
$$

The loading-unloading conditions are

$$
\phi(\boldsymbol{\sigma}, \bar{\epsilon}, f) \leqslant 0 ; \quad \Lambda \geqslant 0 ; \quad \Lambda \phi(\boldsymbol{\sigma}, \bar{\epsilon}, f)=0,
$$

where, for plastic loading, the plastic multiplier $\Lambda$ in Eq. (12) is

$$
\Lambda=\frac{\mathbf{p}: \dot{\boldsymbol{\sigma}}}{h},
$$

where

$$
h=-\left[(1-f) \frac{\partial \phi}{\partial f} \operatorname{tr}(\mathbf{p})+\left(\frac{\partial \phi}{\partial f} \mathcal{A}_{m}+\frac{\partial \phi}{\partial \bar{\sigma}} \bar{h}\right) \frac{\boldsymbol{\sigma}: \mathbf{p}}{(1-f) \bar{\sigma}}\right]
$$

with

$$
\bar{h}=\frac{d \bar{\sigma}}{d \bar{\epsilon}} .
$$

In Eq. (20), the coaxiality between $\mathbf{p}$ and $\boldsymbol{\sigma}$ assures the frame-invariant property of the product $(\mathbf{p}: \dot{\boldsymbol{\sigma}})$.

By following a standard procedure, where the plastic strain rate tensor, $\mathbb{d}^{p}$, defined in Eq. (12), is substituted into Eq. (8) and using the consistency condition, $\phi \equiv 0$ during plastic loading, gives the expression relating the stress convective rate

$$
\mathcal{L}(\tau)=\dot{\tau}-\boldsymbol{l} \tau-\tau \boldsymbol{l}^{T}
$$

\section{and strain rate $\boldsymbol{d}$, through}
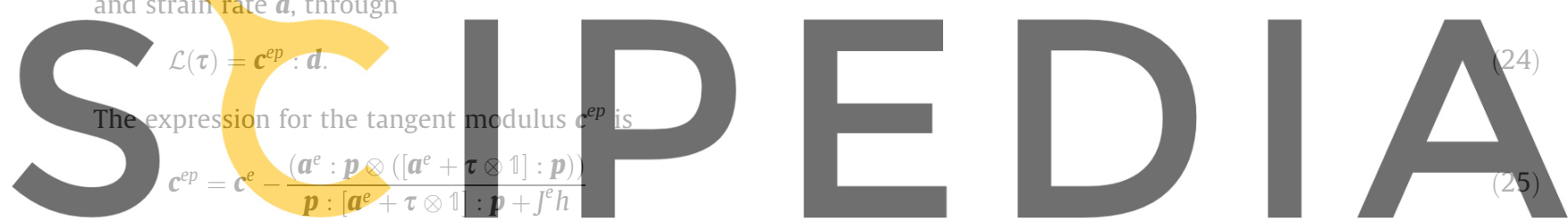

with

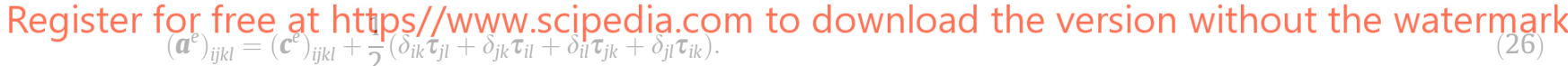

Here, $\boldsymbol{a}^{e}$ is the modified elastic moduhi tensor relating the Jaumann rate of the Kirchhoff stress (defined through the total spin) with the elastic strain rate tensor, $\delta_{i k}$ is the Kronecker delta and indices $i, j=1,2$.

Note that in general $\boldsymbol{c}^{e p}$ is not symmetric since the flow potential, Eq. (13), is expressed in terms of the Cauchy stress and volume changes do not necessarily remain small because of void nucleation and growth.

\subsection{Material bifurcation condition}

As shown by Hadamard (1903), Hill (1962), Rice (1976), a bifurcation corresponding to localization of deformation occurs when the determinant of the acoustic tensor vanishes.

A body $\Omega$ is divided into two parts: $\Omega^{+}$and $\Omega^{-}$by the material surface $\mathcal{S}$ with unit normal vector $\boldsymbol{N}$ in the reference configuration. We analyze the mechanical conditions which permit the possibility of a discontinuous deformation gradient rate through $\mathcal{S}$, given by $\dot{\boldsymbol{F}}^{+}-\dot{\boldsymbol{F}}^{-}=\llbracket \dot{\boldsymbol{F}} \rrbracket=\mathbf{g} \otimes \boldsymbol{N}$, with $\mathbf{g}$ an arbitrary velocity vector, and such that $\dot{\boldsymbol{F}}^{-}=\dot{\overline{\boldsymbol{F}}}$ is defined in $\Omega^{-}$and $\dot{\boldsymbol{F}}^{+}=\tilde{\boldsymbol{F}}+\mathbf{g} \otimes \boldsymbol{N}$ in $\Omega^{+}$.

The equilibrium condition on $\mathcal{S}$ requires the rate of the traction vector, $\dot{\boldsymbol{t}}=\dot{\boldsymbol{P}} \boldsymbol{N}$, with $\boldsymbol{P}=\tau \boldsymbol{F}^{-T}$ the first Piola-Kirchhoff stress tensor, to be continuous across $\mathcal{S}$ :

$$
\llbracket \dot{\boldsymbol{i}} \rrbracket=\llbracket \dot{\boldsymbol{P}} \rrbracket \boldsymbol{N}=\llbracket(\mathcal{L}(\boldsymbol{\tau})+\boldsymbol{l} \tau) \rrbracket \overline{\boldsymbol{e}}=\mathbf{0},
$$

where, we have used the identity $\overline{\boldsymbol{e}}=\left(\boldsymbol{F}^{-}\right)^{-T} \boldsymbol{N}=\left(\boldsymbol{F}^{+}\right)^{-T} \boldsymbol{N}$. Writing the velocity gradient in $\Omega^{-}$and $\Omega^{+}$, as

$$
\boldsymbol{l}^{-}=\overline{\boldsymbol{l}}=\dot{\overline{\mathbf{F}}} \overline{\boldsymbol{F}}^{-1} ; \quad \boldsymbol{l}^{+}=\overline{\boldsymbol{l}}+\frac{1}{1+\mathbf{g} \cdot \overline{\boldsymbol{e}}}(\overline{\mathcal{L}}(\mathbf{g}) \otimes \overline{\boldsymbol{e}}) ; \quad \overline{\mathcal{L}}(\mathbf{g})=\dot{\mathbf{g}}-\overline{\mathbf{l g}},
$$

where the convective rate of the vector $\mathbf{g}$ is denoted $\overline{\mathcal{L}}(\mathbf{g})$. Substituting the constitutive relation Eq. (24) into Eq. (27) and assuming continued plastic loading in both $\Omega^{+}$and $\Omega^{-}$so that $\left(\boldsymbol{c}^{e p}\right)^{+}=\left(\boldsymbol{c}^{e p}\right)^{-}=\boldsymbol{c}^{e p}$, we obtain 


$$
[\underbrace{\overline{\boldsymbol{e}} \cdot \boldsymbol{c}^{e p} \cdot \overline{\boldsymbol{e}}+(\overline{\boldsymbol{e}} \tau \overline{\boldsymbol{e}}) \mathbb{1}}_{\mathbf{Q}^{e p}}] \cdot \overline{\mathcal{L}}(\mathbf{g})=\mathbf{0},
$$

which expresses the condition that a non-null vector $\overline{\mathcal{L}}(\mathbf{g})$ exists only if the acoustic tensor $\mathbf{Q}^{e p}(\overline{\boldsymbol{e}})$ is singular.

\section{Weak discontinuity band kinematics}

The kinematical description of a displacement field with a weak discontinuity is given in Hadamard (1903), Hill (1962), and Rice (1976). In Huespe et al. (2009), the kinematical description was modified to allow for a finite width band, but limited to the context of small deformation theory. Here, we provide the generalization to finite deformations for finite width bands.

A displacement field $\boldsymbol{u}$ with a discontinuity in the deformation gradient field (weak discontinuity) across a material band $\Omega_{0}^{D}$ is considered. The band has finite width $D_{0}$ in the reference configuration, as shown in Fig. 1(a). For simplicity, we assume that $\Omega_{0}^{D}$ is bounded by two parallel straight lines. This displacement field representation admits strain localization, into this finite band width.

In the figure, $\boldsymbol{N}$ is the unit orthogonal vector to the mean surface $\mathcal{S}_{0}$ of $\Omega_{0}^{D}$. The complementary part $\Omega \backslash \Omega_{0}^{D}$ of the body, is divided in two domains, which are denoted: $\Omega^{+}$(in the positive direction of $N$ ) and $\Omega^{-}$.

A displacement field with weak discontinuities can be written as

$$
\boldsymbol{u}(\boldsymbol{X}, t)=\overline{\boldsymbol{u}}(\boldsymbol{X}, t)+\mathcal{H}_{D}(\boldsymbol{X}, t) \boldsymbol{\beta},
$$

where $\boldsymbol{u}(\boldsymbol{X}, t)$ is a smooth displacement component and the second term accounts for the jumps in the displacement gradient field. The function $\mathcal{H}_{D}(\boldsymbol{X}, t)$ is defined as

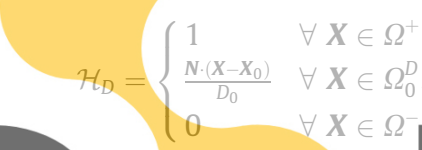

\section{with $D_{0}$ being the initial width} .
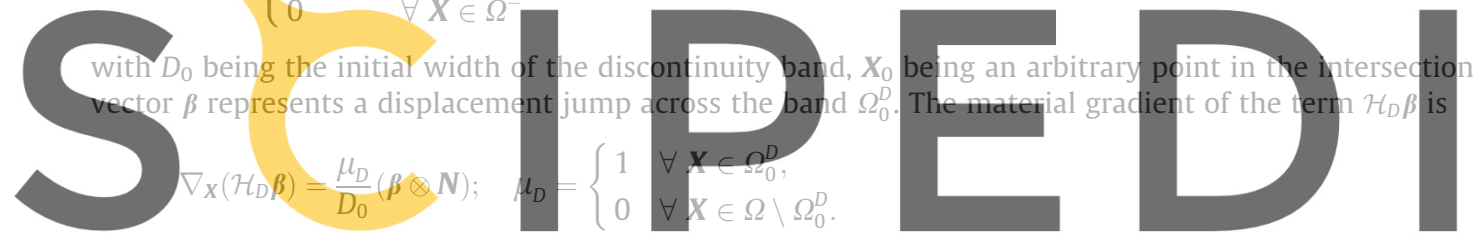

Hence, the deformation gradient can be written in the form (see Fig. 2 (a))

Register for free at https//www.scipedia.com to download the version without the watermark

$$
\begin{aligned}
& \widetilde{\boldsymbol{F}}=\left(\mathbb{1}+\mu_{D} \frac{\beta \otimes \bar{e}}{D_{0}}\right), \\
& \overline{\boldsymbol{F}}=\mathbb{1}+\nabla_{\boldsymbol{X}} \overline{\boldsymbol{u}},
\end{aligned}
$$

which can be seen as a multiplicative decomposition of the deformation gradient (Armero and Garikipati, 1996), defining an intermediate configuration given by the deformation map $\bar{\varphi}(\boldsymbol{X}, t): \Omega_{0} \rightarrow \bar{\Omega}$, whose displacement field is $\overline{\boldsymbol{u}}(\boldsymbol{X}, t)$ and deformation gradient: $\overline{\boldsymbol{F}}$. The orthogonal vector to the discontinuity surface, $\overline{\boldsymbol{e}}$, in the intermediate configuration, $\bar{\Omega}$, is

$$
\overline{\boldsymbol{e}}=\overline{\boldsymbol{F}}^{-T} \boldsymbol{N}
$$

With these expressions, it can be shown that the finite band width, $D_{t}$, in the current configuration is given by

$$
D_{t}=D_{0}+\boldsymbol{\beta} \cdot \overline{\boldsymbol{e}} .
$$

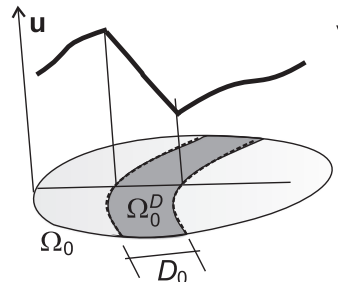

(a)

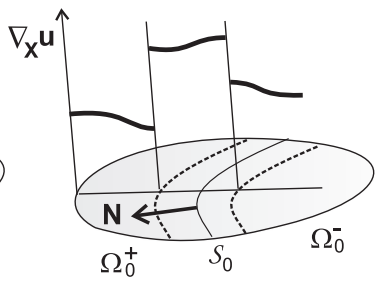

(b)

Fig. 1. Weak discontinuity kinematics. (a) The displacement field. (b) The gradient of the displacement field. 


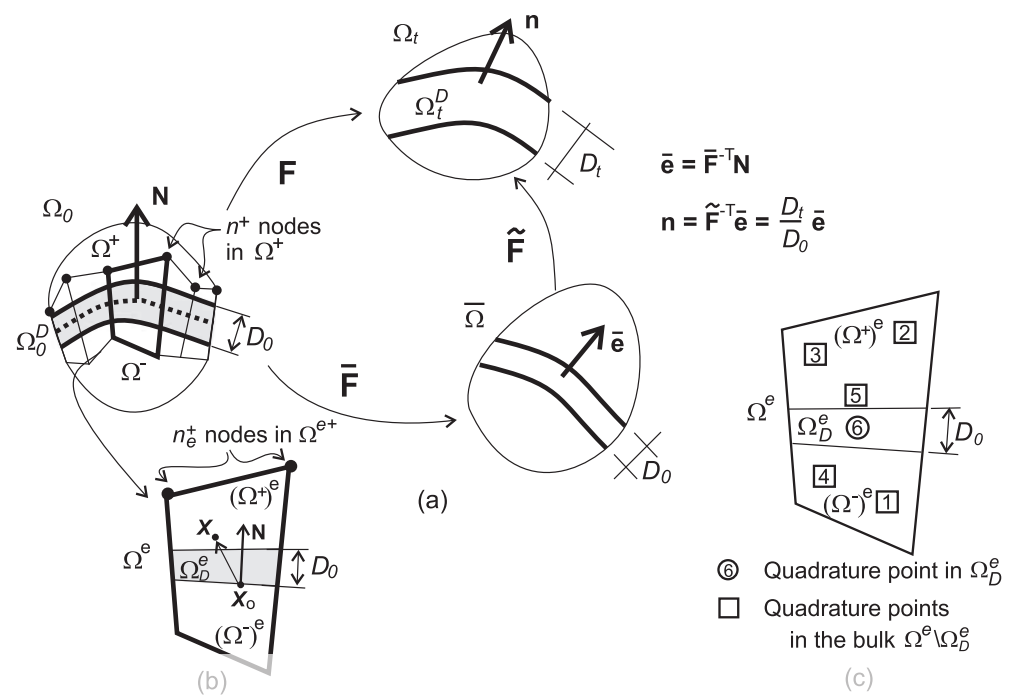

Fig. 2. Finite deformation weak discontinuity kinematics. (a) The multiplicative decomposition of the deformation gradient. (b) A quadrilateral finite element with an embedded weak discontinuity. (c) Quadrature points within a quadrilateral finite element.

ron

Thus, a localized deformation band representing a shear band is described by a deformation mode having displacement jumps, $\beta$, orthogonal to $\bar{e}$. Once formed, the discontinuity band width remains unchanged and the deformation state within
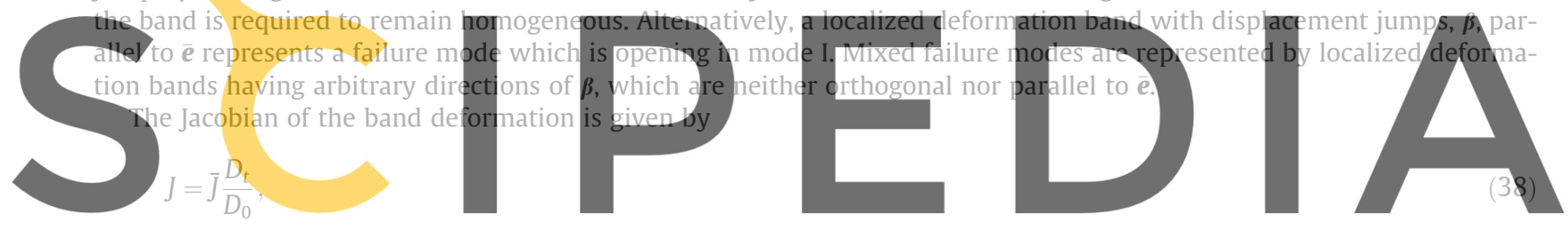

where $\bar{J}=\operatorname{det}(\overline{\boldsymbol{F}})$

Register for free at https//www.scipedia.com to download the version without the watermark

\section{Finite element model}

A number of finite element techniques have been proposed in the past specially designed to simulate strain localization and fracture problems. Basically, these techniques add one enriched kinematic mode to a underlying finite element, that allows for capturing weak discontinuities, such as in Ortiz et al. (1987), Dvorkin et al. (1990), or strong discontinuities, such as in Belytschko et al. (2001), Alfaiate et al. (2003), Armero and Garikipati (1996), Oliver (1996), etc. A recent review of two finite element classes applicable to material modelling problems involving strong discontinuities, such as fracture, is reported in Belytschko et al. (2009).

The numerical procedure we adopt here is based on a finite element methodology with embedded weak discontinuities that closely follows the approach presented in Huespe et al. (2009) for small deformations. As in Huespe et al. (2009) the implementation is carried out for two dimensional problems.

The discretization of the reference configuration $\Omega_{0}$, is performed by means of a quadrilateral finite element mesh. In Fig. 2(b), a finite element is denoted $\Omega^{e}$ and the intersection of the localization band with this finite element is denoted $\Omega_{D}^{e}$. The complementary parts of the finite element are denoted by $\left(\Omega^{+}\right)^{e}$ and $\left(\Omega^{-}\right)^{e}$.

In each finite element, the displacement field (30) is interpolated by

$$
\boldsymbol{u}(\boldsymbol{X}, t)=N_{i}(\boldsymbol{X}) \boldsymbol{q}_{i}+\mathcal{M}_{D}^{e}(\boldsymbol{X}) \boldsymbol{\beta}^{e},
$$

where $N_{i}(\boldsymbol{X}), i=1,2, \ldots, 4$ are the standard bilinear quadrilateral shape functions and $\boldsymbol{q}_{i}$ are the nodal displacements. The second term accounts for the deformation gradient discontinuity, and is written as

$$
\mathcal{M}_{D}^{e}(\boldsymbol{X}) \boldsymbol{\beta}^{e}=\left(\mathcal{H}_{D}^{e}(\boldsymbol{X})-N^{s o l}(\boldsymbol{X})\right) \boldsymbol{\beta}^{e},
$$

where $\mathcal{H}_{D}^{e}(\boldsymbol{X})$ is defined in a similar way as the expression in Eq. (31), restricted to the element domain. Also, $\left.N^{\text {sol }}(\boldsymbol{X})\right)$ is the addition of the shape functions $N_{i}$ associated to the element nodes $\left(n_{e}^{+}\right)$lying in $\left(\Omega^{+}\right)^{e}$. The addition of the term $N^{\text {sol }}(\boldsymbol{X})$ facilitates the imposition of natural boundary conditions in the discrete boundary value problem. 
In the approximation of the displacement enriching mode defined by (40), $\boldsymbol{\beta}^{e}$ is a vectorial parameter, constant in the finite element, which allows for an inexpensive element-wise condensation of these degrees of freedom.

In order to avoid the numerical locking effects due to possible near isochoric deformations in the plastic regime, we adopt an underlying finite element which is based on a strain enhanced methodology, such as that proposed by Simo (1998).

\subsection{Discrete equilibrium equations}

The variational equilibrium Eq. (1) can be discretized using a Petrov-Galerkin approach, see Oliver et al. (2003), which can be written as

$$
\begin{aligned}
& \int_{\Omega_{0}} \boldsymbol{B}_{u}^{T} \boldsymbol{\sigma} J d \Omega_{0}=\boldsymbol{G}^{e x t}, \\
& \frac{1}{\Omega_{D}^{e}} \int_{\Omega_{D}^{e}} \boldsymbol{\sigma} \cdot\left(\boldsymbol{F}^{-T} \boldsymbol{N}\right) J d \Omega_{D}^{e}-\frac{1}{\Omega_{c}^{e}} \int_{\Omega_{c}^{e}} \boldsymbol{\sigma} \cdot\left(\boldsymbol{F}^{-T} \boldsymbol{N}\right) J d \Omega_{c}^{e}=\mathbf{0},
\end{aligned}
$$

where $\boldsymbol{G}^{\text {ext }}$ is the discrete external force and $\boldsymbol{B}_{u}$ is a spatial matrix representing the linearized strain-displacement operator, and is defined according to the enhanced finite element formulation of Simo (1998). Eq. (42) is elementwise imposed only in those elements where embedded localization bands have been introduced. In the second term of that equation, the integration domain, $\Omega_{c}^{e}$, is the complementary part of the embedded discontinuity band: $\Omega_{c}^{e}=\Omega^{e} \backslash \Omega_{D}^{e}$.

Eq. (41) is standard in finite element procedures, while Eq. (42) imposes a weak force balance across the discontinuity band.

4.2. Algorithmic implementation details

Specific algorithmic aspects in the present context of large deformation kinematics, are noted here.

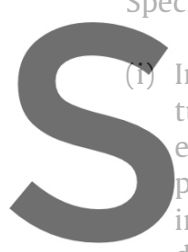

In each finite element, the ture points, see Fig. 2(c). element. The fifth and six points 1 to 5 represent the in Section 3, and the sixth Gauss point represents the deformation gradient at a regular quadrature point $M$ is denoted by $\mathbb{F}_{M}$, with indices $M$ ranging from 1 to 4 , and evaluated through

Register for free $F_{F_{M}}=\int_{0} F_{M}$, https//www.scipedia.com to download the version without the watermark

where $J_{0}$ is the determinant of the deformation gradient at the element central point, the fifth quadrature point, and $\boldsymbol{F}_{M}^{\text {iso }}=J_{M}^{-(1 / 3)} \boldsymbol{F}_{M}$, such that $\operatorname{det} \boldsymbol{F}_{M}^{\text {iso }}=1$. The stress tensor at quadrature point $M, \boldsymbol{\sigma}_{M}$, is determined by the deformation gradient $\boldsymbol{F}_{M}$ using the constitutive relation in SubSection 2.2. Integration of Eq. (41) is carried out using the standard Gauss points 1 to 4, and the matrix $\boldsymbol{B}_{u}$ is defined according to the enhanced finite element formulation of Simo (1998). Eq. (42) is evaluated point-wise, by using the fifth and sixth integration points.

(ii) The determinant of the acoustic tensor in Eq. (29) is evaluated in the current configuration at the central integration point of every finite element. The localization mode is activated when the acoustic tensor becomes singular. The corresponding eigenvectors give two possible localization directions. Once localization initiates in some finite element for some unit vector $\overline{\boldsymbol{e}}_{0}$, the enriching mode in Eq. (39) is activated in that element. The numerical detection of the singularity of the acoustic tensor, is determined using the iterative algorithm in Oliver et al. (2010).

Once determined $\overline{\boldsymbol{e}}_{0}$, its normalized pull back transformation to the reference configuration is then given by $\boldsymbol{N}=\overline{\boldsymbol{F}}^{T} \overline{\boldsymbol{e}}_{0} /\left(\left\|\overline{\boldsymbol{F}}^{T} \overline{\boldsymbol{e}}_{0}\right\|\right)$. With $\boldsymbol{N}$ defined in every point of this configuration, we trace the complete set of possible discontinuity paths according to the procedure reported in Oliver et al. (2004). This procedure evaluates the envelopes of the tangent vector field $\boldsymbol{T}$, orthogonal to $\boldsymbol{N}$, and requires the definition of a unique normal vector for every material point. However, eq. (29) provides, in general, two eigenvectors at each point. Both eigenvector solutions may be associated with different, and admissible, localization modes. We have not yet developed a general criteria for selecting one specific eigenvector solution for evaluating the crack path. Therefore, in the calculations a heuristic criterion is used to obtain the localization direction.

(iii) An Impl-Ex scheme, see Oliver et al. (2008), Sánchez et al., 2008, is used in order to integrate, in time, the evolution equations of the problem. Box 1 present a sketch of the proposed scheme, where $\Delta \boldsymbol{u}_{n+1}$ is the incremental displacement at step $(n+1)$. After evaluating trial values, Point 1 in the Box, two sequence of variables are calculated per time step: (1) an implicit sequence (denoted with the symbol: $(\hat{)})$ ) which is presented in Point 2 of the Box; and (2) a semiexplicit sequence (denoted with the symbol: $(\tilde{)})$ ) and presented in Point 3 of the Box. The Jacobian of the elastic deformation of the previous step $\left(J_{n}^{e}\right)$ is used in Point 1.4 to calculate the trial Cauchy stress, and remain fixed during the 


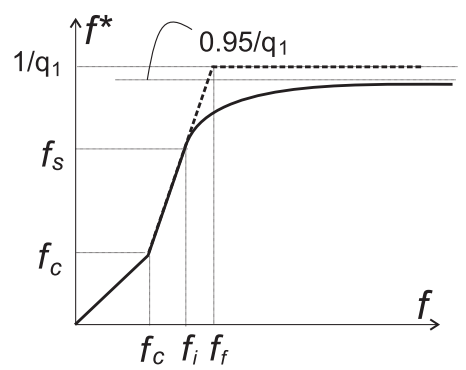

Fig. 3. The function $f^{*}$ in Eq. (44) compared with the bilinear expression in Eq. (15).

iterative process for evaluating the implicit Cauchy stress in Point 2.2. The Impl-Ex Cauchy stress (Points 3.3 and 3.4) is calculated by using the principal directions of $\boldsymbol{V}_{n+1}^{\text {trial }}$, while the principal values are obtained through a particularization of the procedure presented in the Point 3, Box 2 of Sánchez et al. (2008). This Impl-Ex Cauchy stress is used to evaluate the discrete equilibrium Eqs. (41) and (42). With this procedure, the resulting Impl-Ex stresses do not exactly satisfy the plastic consistency condition and remain a non-linear function of the displacements. However, due to the explicit extrapolation of internal variables, the algorithmic tangent tensor resulting from the integration in Box 1 is positive definite, and therefore, the characteristic robustness of the Impl-Ex scheme is preserved.

(iv) In those elements where the void fraction value becomes larger than a specified value $f_{x}$, the values of the void volume fraction $f$ and the matrix plastic strain $\bar{\epsilon}$ are kept fixed. Typically, at least in the calculations we have carried out, this occurs inside a localization band, i.e. at the sixth quadrature point of a finite element with an embedded discontinuity mode. Thus, once $f>f_{x}$, the yield function remains unchanged forcing the stresses on this surfaces to be close to zero. Therefore, there is almost no contribution from this quadrature point to the internal nodal forces. With this approach finite elements do not need to be removed from the mesh during the course of the numerical simulation. We have taken $f_{x}=0.55$

The function $f^{*}$, defined upper limit of the void $f$
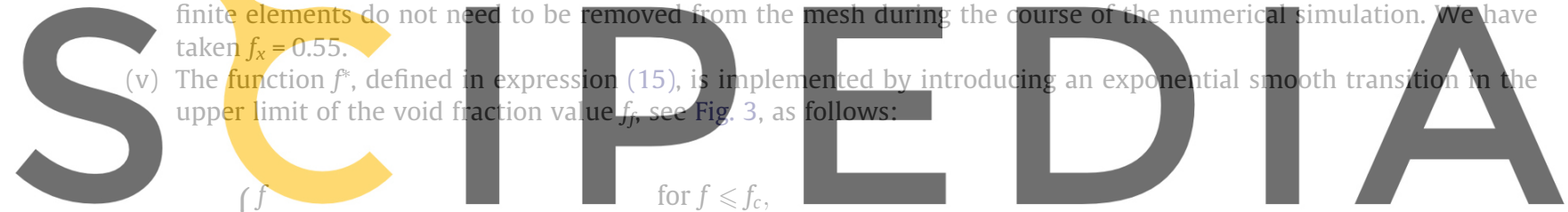

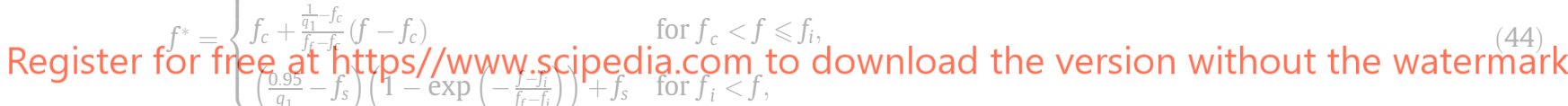

where $f_{i}$ is the value of $f$ at which the transition from the linear function to the exponential one takes place, $f_{i}=0.75\left(f_{f}-f_{c}\right)+f_{c}$ and $f_{s}$ is the value $f^{*}\left(f_{i}\right)$ given by the second of Eq. (15).

In this way, $f \in[0, \infty)$, and we can preserve the numerical treatment described in item (iv), when $f>f_{x}$. A similar smoothing of the function $f^{*}(f)$ was used in Mathur et al. (1994).

\section{Numerical examples}

Numerical examples are presented to illustrate the applicability of the methodology in cases exhibiting a variety of failure modes. The examples considered are: (i) plane strain tension of a rectangular bar leading to a shear failure, SubSection 5.1; (ii) axisymmetric tension of a cylindrical bar giving a cup-cone failure, SubSection 5.2; and (iii) plane strain tension of a notched bar where failure involves a shear mode that changes direction, SubSection 5.3. In several cases, results for different mesh resolutions and for different values of the band thickness $D_{0}$ are compared. Also, in some cases the results obtained are compared with previous solutions taken from the literature.

\subsection{Plane strain tension}

In plane strain tension, the predicted failure mode with porous plasticity is a shear band mode, see for example Becker and Needleman (1986), Besson et al. (2003). Here, we carry out the calculations for the rectangular bar sketched in Fig. 4(a) with $L / e_{m}=3.5$. A geometrical imperfection is introduced to induce the initiation of the localization band to lie in the bar central zone. It consists of a linear decrease of the bar net section with a maximum reduction area of $2 \%\left(e_{0}=0.98 e_{m}\right)$. The width $e_{0}$ defines the reference area in the following results. 
Box 1: The Impl-Ex numerical integration algorithm.

Integration step: $(n+1)$ with time increment: $\left(\Delta t_{n+1}\right)$

GIVEN: $\boldsymbol{F}_{n} ; \Delta \boldsymbol{u}_{n+1} ;$ and the implicit values of the previous step: $\widehat{\boldsymbol{F}}_{n}^{e} ; \widehat{\boldsymbol{F}}_{n}^{p} ; \hat{\bar{\epsilon}}_{n} ; \hat{f}_{n}$

\section{(1) Find trial values:}

(1.1) $\boldsymbol{F}_{n+1}^{\text {trial }}=\mathcal{F}\left(\Delta \boldsymbol{u}_{n+1}\right) \hat{\boldsymbol{F}}_{n}^{e} ; \quad \mathcal{F}\left(\Delta \boldsymbol{u}_{n+1}\right)=1+\frac{\partial \Delta \boldsymbol{u}_{n+1}}{\partial \boldsymbol{x}_{n}}$

(1.2) $\boldsymbol{V}_{n+1}^{\text {trial }}=\left[\boldsymbol{F}_{n+1}^{\text {trial }}\left(\boldsymbol{F}_{n+1}^{\text {trial }}\right)^{T}\right]^{1 / 2}$ and its spectral decomposition:

(1.3) $\boldsymbol{V}_{n+1}^{\text {trial }}=\sum_{I=1}^{3} \lambda_{I}^{\text {trial }}\left(\boldsymbol{r}_{I}^{n+1} \otimes \boldsymbol{r}_{I}^{n+1}\right)$ Evaluate the trial Cauchy stress using Eq. (7)

(1.4) $\boldsymbol{\sigma}^{\text {trial }}=\sum_{I=1}^{3} \sigma_{I}^{\text {trial }}\left(\boldsymbol{r}_{I}^{n+1} \otimes \boldsymbol{r}_{I}^{n+1}\right)$ where: $\sigma_{I}^{\text {trial }}=\frac{1}{J_{n}^{e}}\left(\frac{E}{1+v}\left[\ln \left(\lambda_{I}^{\text {trial }}\right)+\frac{v}{1-2 v}\left(\sum_{K=1}^{3} \ln \left(\lambda_{K}^{\text {trial }}\right)\right)\right]\right)$

(2) In the space of principal stresses, evaluate the following implicit values: (use a return mapping scheme, similar to that of BOX 1 in Sánchez et al., 2008)

(2.1) $\widehat{\Lambda}_{n+1} ;\left(\hat{\sigma}_{I}\right)_{n+1} ; \hat{\bar{\epsilon}}_{n+1} ; \hat{f}_{n+1} ; \hat{\boldsymbol{F}}_{n+1}^{e} ; \hat{\boldsymbol{F}}_{n+1}^{p}$; such that:

(2.2) $\left(\hat{\sigma}_{I}\right)_{n+1}=\sigma_{I}^{\text {trial }}-\widehat{\Lambda}_{n+1} \overline{\boldsymbol{c}}_{I J} \partial_{\sigma_{J}} \phi_{n+1}$ and $\phi_{n+1}\left(\left(\hat{\sigma}_{K}\right)_{n+1}, \hat{\bar{\epsilon}}_{n+1} ; \hat{f}_{n+1}\right)=0$; the tensor $\overline{\boldsymbol{c}}$ is defined in Eq. (10) (here expressed in $\mathbb{R}^{3}$ )

(3) Evaluate the Impl-Ex internal variables: (use a scheme, similar to that of BOX 2 in Sánchez et al. (2008))

(3.1) $\tilde{\Lambda}=\widehat{\Lambda}_{n} \frac{\Delta t_{n+1}}{\Delta t_{n}}$;

(3.2) $\tilde{\bar{\epsilon}}_{n+1}=\hat{\bar{\epsilon}}_{n}^{\Delta t_{n}}$

(3.3) $\tilde{f}_{n+1}=\hat{f}_{n}$; and the principal values of the Impl-Ex stress,

(3.3) $\left(\tilde{\sigma}_{l}\right)_{n+1}$; Evaluate Impl-Ex stress:

(3.4) $\widetilde{\boldsymbol{\sigma}}_{n+1}=\sum_{I=1}^{3}\left(\widetilde{\sigma}_{I}\right)_{n+1}\left(\boldsymbol{r}_{I}^{n+1} \otimes \boldsymbol{r}_{I}^{n+1}\right)$

OUTPUT: $\widehat{\boldsymbol{F}}_{n+1}^{e} ; \widehat{\boldsymbol{F}}_{n+1}^{p} ; \hat{\bar{\epsilon}}_{n+1} ; \hat{f}_{n+1} ; \widetilde{\boldsymbol{\sigma}}_{n+1}$
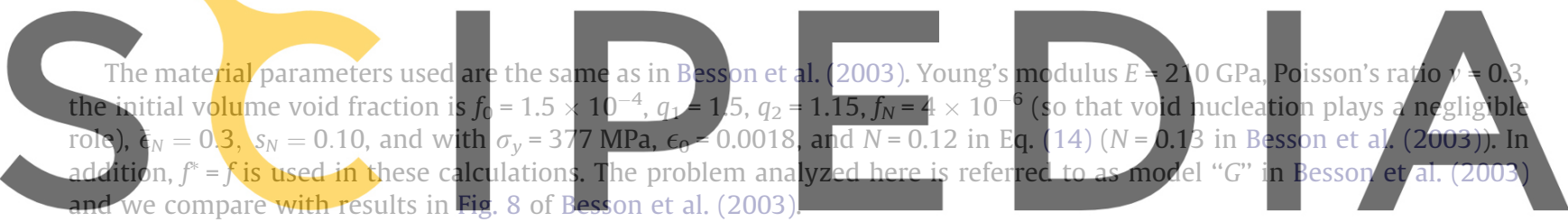

Three quadrilateral finite element Meshes 1, 2 and 3, as shown in Fig. 4(c), are used. They have a uniform distribution of

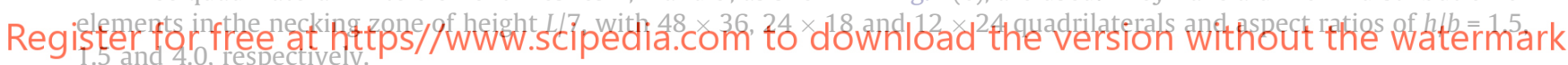

The deformed finite element meshes together with the void fraction distributions are shown in Fig. 4(c) at the end of the analysis. A very similar shear band mode occurs for all three meshes. There are two possible localization band directions. At the central point of the bar, the angles between the critical localization directions and the horizontal symmetry axis are $\pm 44.5^{\circ}$ in the deformed configuration, which are in agreement with those reported in Besson et al. (2003). In the reference configuration, these angles are $\pm 12.5^{\circ}$. In the calculations here, we arbitrarily choose to introduce one of these possible band orientations.

Fig. 4(b) shows the void volume fraction distribution, in the deformed configuration, when the first bar material point satisfies the bifurcation condition.

Overall nominal traction (force $F /$ unit reference area $S_{0}$, with $S_{0}=2 e_{0}$ assuming unit thickness), versus the area reduction at the minimum section $|\Delta e| / e_{0}$ is shown in Fig. $4(\mathrm{~d})$ for the three finite element meshes with $D_{0} / e_{0}=0.0033$. The results are compared with those in Besson et al. (2003). The overall responses even after localization are rather independent of the number of finite elements in the mesh. Although the widths of the regions with a high void volume fraction $f$ in Fig. 4(c) clearly depend on the mesh resolution, in each case the main dissipation actually occurs with a localization band of width $D_{0}$ so that the dissipation is relatively mesh independent. Fig. 4(e) illustrates the dependence of the response on the band width $D_{0}$ with a fixed finite element mesh. The post-localization response does depend on $D_{0}$ since the band width determines the dissipation during localization.

Fig. 5 compares the solution obtained with an unstructured mesh with that obtained using the structured Mesh 1 having 48 finite elements across the bar width. The overall traction-area reduction curves essentially coincide, Fig. 5(a). Figs. 5(b) and (c) show the shear band orientation in the deformed and undeformed unstructured meshes, respectively. The band orientation in Fig. 5(b) is in very good agreement with those seen in Fig. 4(c).

\subsection{Axisymmetric tension}

In contrast to plane strain, the predicted failure mode for a porous plastic solid in axisymmetric tension is a cup-cone mode, as is also seen experimentally, Tvergaard and Needleman (1984) and Besson et al. (2001). We carry out calculations 


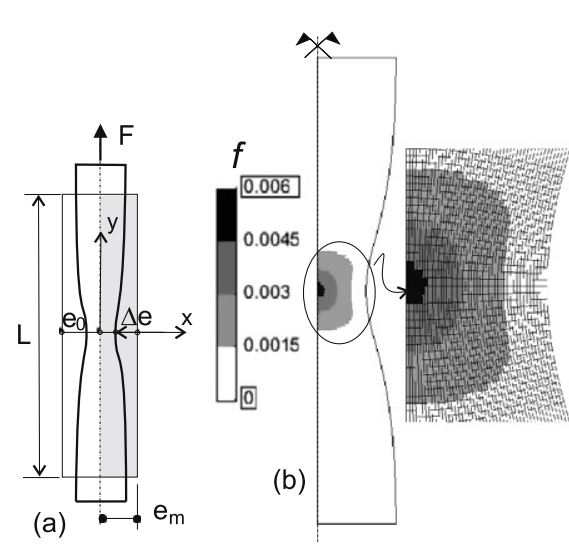

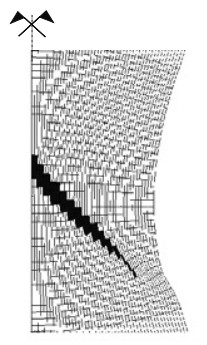

Mesh 1 (48 FE)

$\mathrm{h}=\mathrm{e}_{\mathrm{o}} / 48$

$\mathrm{h} / \mathrm{b}=1.5$

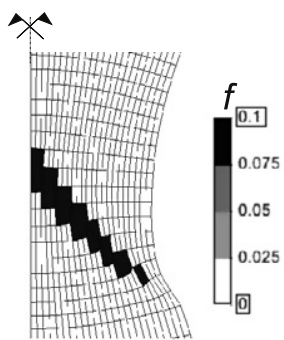

Mesh 2: (24 FE)

$\mathrm{h}=\mathrm{e}_{\mathrm{o}} / 24$

$\mathrm{h} / \mathrm{b}=1.5$

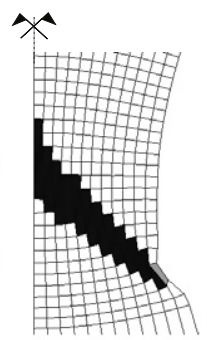

Mesh 3: (12 FE)

$\mathrm{h}=\mathrm{e}_{\mathrm{o}} / 12$

$h / b=4$.

(c)
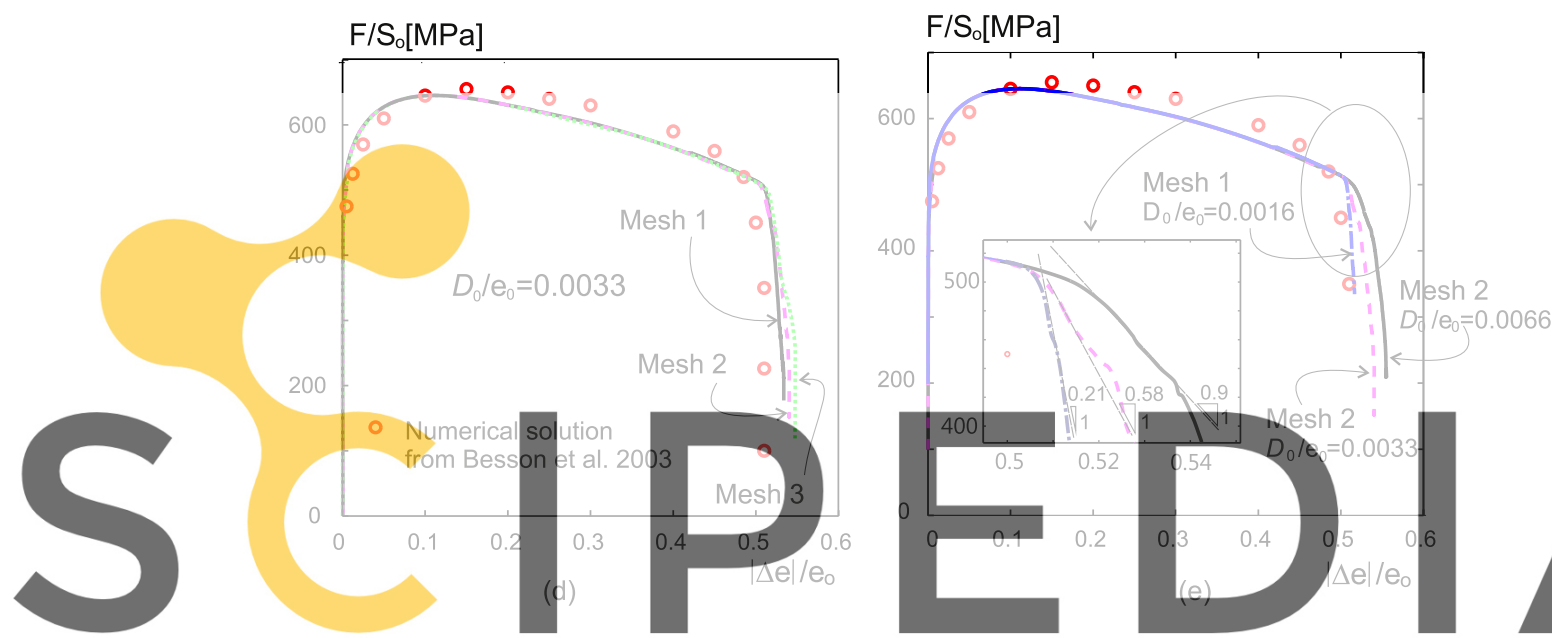

Fig. 4. Localization and failure in a plane strain specimen. (a) A sketch illustrating the boundary value problem. (b) Void volume fraction distribution, in the deformed configuration, when the first material point (which is at the specimen center) satisfies the bifurcation condition. (c) Contours of void volume

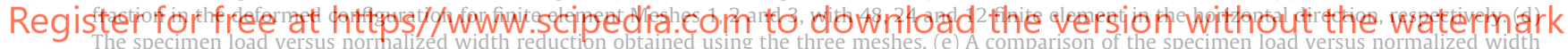
reduction curves obtained with three values of band width $D_{0}$.

of axisymmetric tensile specimens for two cases; in one case there is an initial volume fraction of voids with a low volume fraction of void nucleating particles, as considered by Besson et al. (2001), and with $f^{*}=f$. In the other case, there are no initial voids, $f_{0}=0$, and the volume fraction of void nucleating particles is much larger. Also, the void coalescence function $f^{*}$ is used as in Tvergaard and Needleman (1984) but with the modification of Eq. (44).

We first consider the case with a low volume fraction of void nucleating particles $f_{N}=4 \times 10^{-6}$ and with $f_{0}=1.5 \times 10^{-4}$, as in Besson et al. (2001). Other material parameters are: Young's modulus $E=210 \mathrm{GPa}$, Poisson's ratio $v=0.3, q_{1}=1.5, q_{2}=1.15, \bar{\epsilon}_{N}=0.3, s_{N}=0.10, \sigma_{y}=377 \mathrm{MPa}, \epsilon_{0}=0.0018$, and $N=0.12$. Fig. $6(\mathrm{a})$ shows the specimen analyzed which has an initial specimen length, $L$, to radius, $r_{m}$, ratio of $L / r_{m}=3.5$. A geometrical imperfection is introduced to induce the initiation of the localization band to lie in the central zone of the bar. The geometrical imperfection consists of a decrease of the bar net section, in the central zone of height $L / 7$, following a circular profile as shown in Fig. 6(a), with a maximum radius reduction: $r_{0}=0.98 r_{m}$. In the following analysis, the reference bar radius is $r_{0}$.

The quadrilateral finite element meshes used are shown in Fig. 6(b). Mesh 1 has $35 \times 95$ uniform elements in the neck region, of height $L / 7$, and a total of 3985 quadrilateral elements; Mesh 2 has $25 \times 69$ uniform elements in the neck region and a total of 2180 quadrilateral elements; and Mesh 3 has $23 \times 43$ uniform elements in the neck region and a total of 1824 quadrilateral elements. In the neck region, the elements have an initial aspect ratio of $h / b=5.4$, with $h$ and $b$ the element width and height respectively for Meshes 1 and 2 and $h / b=3.7$ for Mesh 3. The material parameters for the case considered in Fig. 6 are identical to those used in the plane strain tension case in SubSection 5.1.

Void volume fraction contours for the three meshes are displayed in the deformed configuration in Fig. 6(b). The dark elements are those with a void volume fraction $f$ greater than 0.2 . Failure initiates at the specimen center and initially propagates along a zig-zag path that is approximately perpendicular to the tensile axis as discussed by Tvergaard and Needleman (1984). Once the failure region extends over about $30 \%$ of the specimen cross section, the failure mode changes to a shear mode giving rise to the final cup-cone failure. The localization bifurcation analysis has two possible (symmetric) shear localization directions and in the calculations one is chosen arbitrarily. 

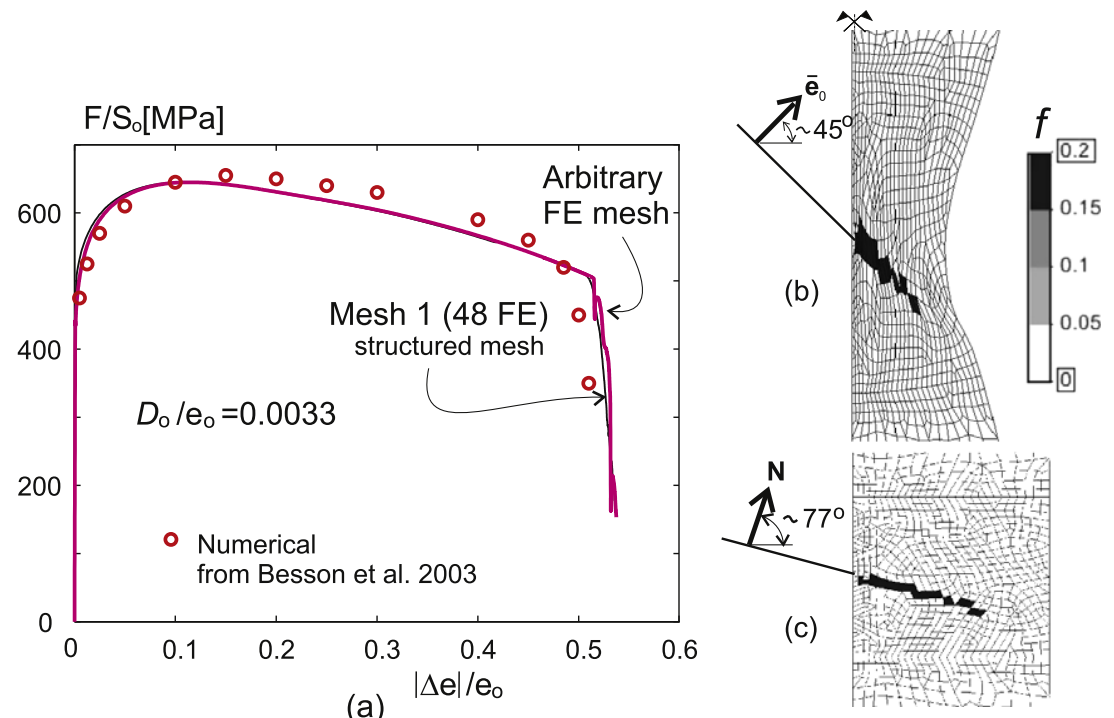

Fig. 5. Localization and failure in a plane strain specimen. (a) Specimen load versus normalized width reduction curves obtained with an unstructured finite element mesh compared with the solution obtained with a structured mesh (Mesh 1 of Fig. 4). (b) Contours of void volume fraction at the end of the analysis and the deformed configuration for the calculation using the unstructured mesh. (c) Contours of void volume fraction in the undeformed configuration for the calculation using the unstructured mesh.

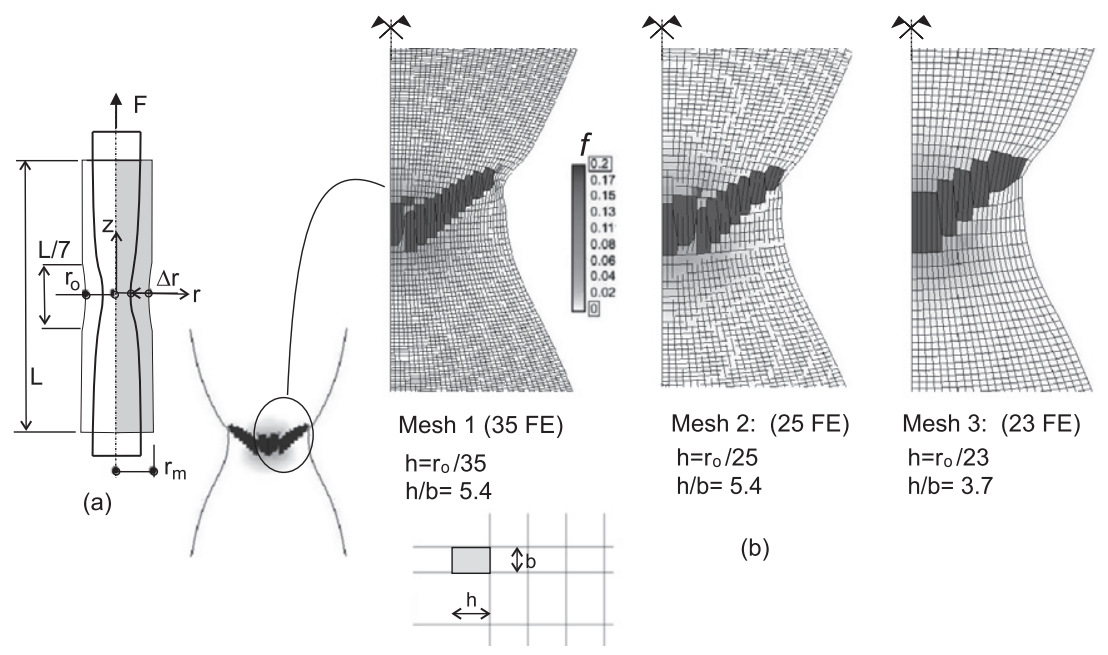

Fig. 6. Development of the cup-cone fracture mode in axisymmetric tension with $f_{N}=4 \times 10^{-6}$. (a) A sketch illustrating the boundary value problem. (b) Finite element meshes and contours of void fraction $f$ at the end of the analysis displaying the fracture surface for three deformed meshes $\left(|\Delta r| / r_{0}=0.518\right)$. The notations (35FE) for Mesh 1, (25FE) for Mesh 2 and (23FE) for Mesh 3 refer to the number of quadrilateral finite elements in the radial direction. The initial element aspect ratio, $h / b$, is also noted.

Figs. 7(a) and (b) show curves of traction (force $F /$ initial cross sectional area $S_{0}$ ) versus magnitude of the radius reduction, $|\Delta r| \mid r_{0}$, at $z=0$. In Fig. 7(a), results are shown for all three finite element meshes with a fixed localization band width of $D_{0} /$ $r_{0}=0.003$. Also, for comparison purposes results from Besson et al. (2001) are shown. Our results have a somewhat larger strain at which failure occurs. In Fig. 7(b), Mesh 2 is used with two values of the localization band width, $D_{0} / r_{0}=0.0033$ and $D_{0} / r_{0}=0.0066$. At least in this case, doubling the band width has only a small effect on the result. The sharp drop in traction occurs when failure initiates at the specimen center and the change in area reduction between the initiation of failure and the complete loss of stress carrying capacity is very small.

The evolution of the fracture process obtained with the present numerical method is shown in Fig. 8. Fig. 8(a) displays the development of the localization bands during the loading process. Every band, which is introduced when the acoustic tensor, Eq. (29), becomes singular at the central point of a finite element, is represented by a different shade of gray, and plots B and $C$ display the formation of bands denoted 1-5 which indicates the order in which they occur. Whenever the failure 


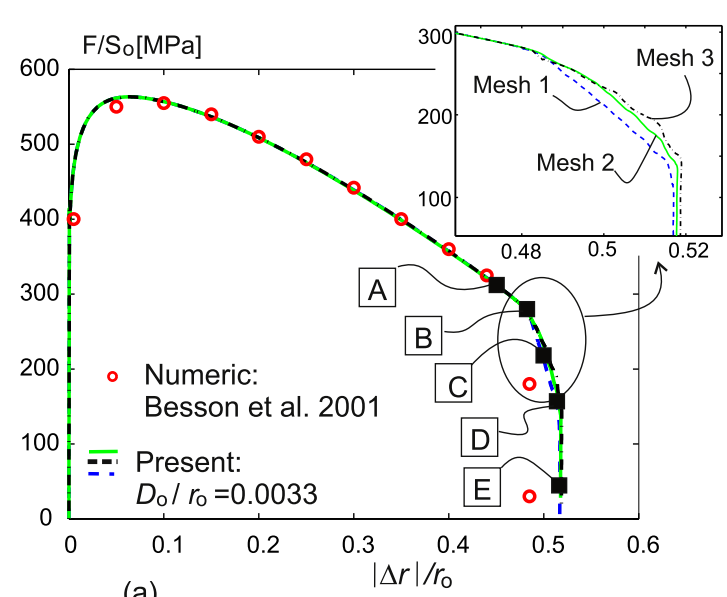

(a)

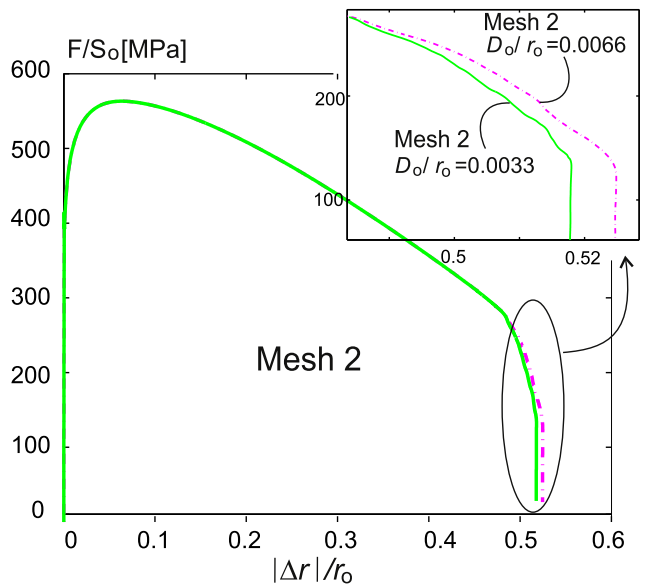

(b)

Fig. 7. Axisymmetric tension with $f_{N}=4 \times 10^{-6}$. (a) Curves of load (Force $F /$ reference area $S_{0}$ ) versus the magnitude of the radius reduction using Meshes 1 , 2 and 3 in Fig. 6 and a localization band width $D_{0} / r_{0}=0.003$. (b) Curves of load versus the magnitude of the radius reduction using Mesh 2 and two localization band widths $D_{0} / r_{0}=0.0033$ and $D_{0} / r_{0}=0.0066$.

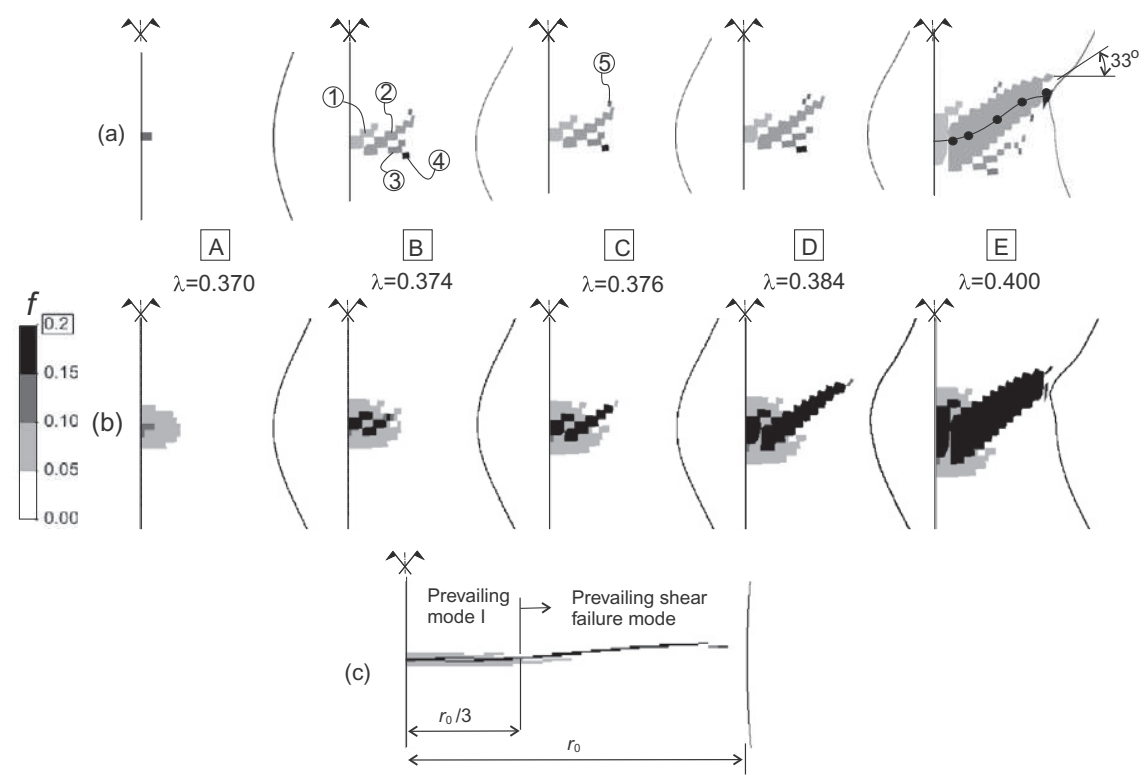

Fig. 8. Axisymmetric tension with $f_{N}=4 \times 10^{-6}$. (a) Development of the localization band pattern during the loading process and the final set of active localization bands (marked with dots) showing the failure path for Mesh 1. The states A, B, C, D and E correspond to the points marked in Fig. 7(a) and $\lambda$ is the logarithm of the average bar stretching, $\lambda=\log (1+\Delta L / L)$. (b) Evolution of the void volume fraction at states A, B, C, D and E. (c) The distribution of void volume fraction at stage $\mathrm{E}$ in the reference configuration.

mechanism involves a zig-zag failure mode, as in the present case, the shear localization band directions should change alternatively during failure propagation. Since we only allow for one of the two possible localization directions, the only possibility for capturing a zig-zag failure mode is by the coalescence of two closely spaced parallel bands. This induces an error because the finite elements lying between parallel bands are not enhanced with the weak discontinuity mode.

As in the plain strain case, there are two possible localization band directions and we arbitrarily choose one of them. In the central point of the bar, the critical angles during the onset of the first localization band, denoted 1 in Fig. 8 (a), are $\pm 21^{\circ}$ between the localization band and the horizontal direction. The critical angle of the second localization band is $\pm 24^{\circ}$, while the final failure has an angle of approximately $\pm 33^{\circ}$ with the horizontal direction, as can be seen in Fig. 8(a), stage E.

Defining a band as active when $\dot{f}>0$ in the band, Fig. 8(b) shows that not all bands remain active during the loading process, such as those denoted by 3, 4 and 5 . The first band, 1 , arrests shortly after its activation. Then, a new band initiates close to the first one, and then coalesces with the arrested band. This process continues with the formation of new localization 
bands leading to an almost horizontal (in an average sense) zig-zag localization band until reaching a fraction of the bar radius where the axisymmetric constraint is relaxed and shear localization occurs. In Fig. 8(c) the contours of void volume fraction $f$ are shown in the reference configuration. this plot shows that failure occurs mainly in a tensile mode for $r<r_{0} / 3$ and mainly in a shear mode for the remainder of the cross section.

We now consider a case with no initial voids, $f_{0}=0$, and a larger volume fraction of void nucleation particle, $f_{N}=0.04$, as in Tvergaard and Needleman (1984). The material parameters used are: $E=210 \mathrm{GPa}, v=0.3 ; q_{1}=1.5, q_{2}=1, \bar{\epsilon}_{N}=0.3$, $s_{N}=0.10, \sigma_{y}=300 \mathrm{MPa}, \epsilon_{0}=\sigma_{y} / E, N=0.1$. We also use Eq. (44) with $f_{c}=0.15, f_{f}=0.25, f_{i}=0.225$ and take $D_{0} / r_{0}=0.0033$.

Fig. 9(a) compares the nominal traction versus the magnitude of the radius reduction at the mid-section for the same three meshes as shown in Fig. 6, namely Meshes: 1, 2 and 3. There is relatively little mesh dependence over the range computed. Fig. 9(b) compares the nominal traction versus the magnitude of the radius reduction at the mid-section using Eq. (44) with a case using the same material parameters but with $f^{*} \equiv f$. As expected, the response of the two cases coincides until the earlier onset of the load drop that takes place when the coalescence expression Eq. (44) is used.

Fig. 10 shows the development of the cup-cone failure mode. Contours of void volume fraction $f$ are shown at the six points marked in Fig. 9(b) for the calculation using Mesh 1. As in the case with a small volume fraction of void nucleating particles, not all localization bands remain active throughout the analysis. Comparing Figs. 8(b) and 10, shows that although

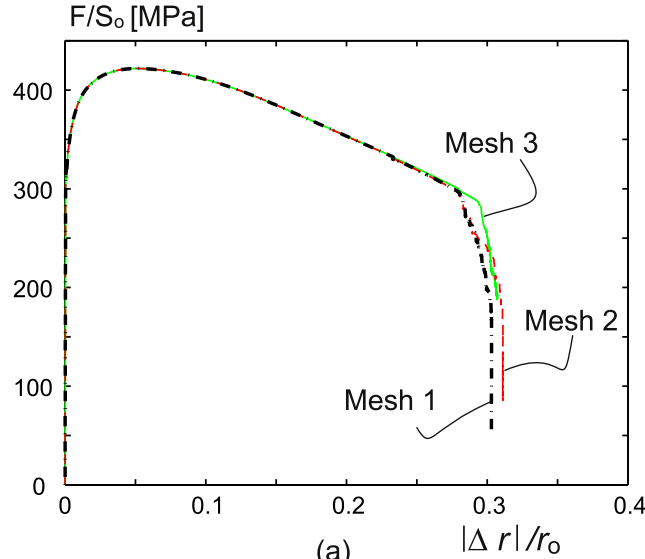

(a)

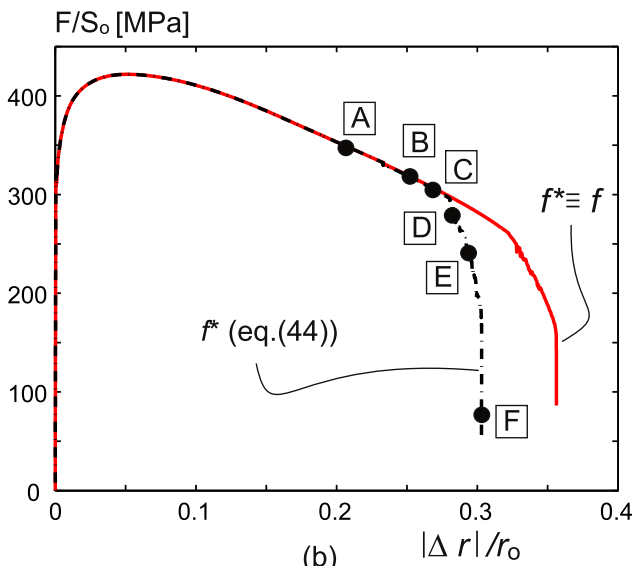

(b)

Fig. 9. Axisymmetric tension with $f_{N}=0.04$. (a) Curves of specimen load versus the magnitude of the radius reduction at the minimum section obtained with Meshes 1, 2 and 3 in Fig. 6(b) using $f *$ from Eq. (44) and with $D_{0} / r_{0}=0.0033$. (b) A comparison of the curves of specimen load versus the magnitude of the radius reduction at the minimum section obtained with Mesh 1 in Fig. 6 (b) using $f^{*}$ in Eq. (44) and using $f^{*} \equiv f$.
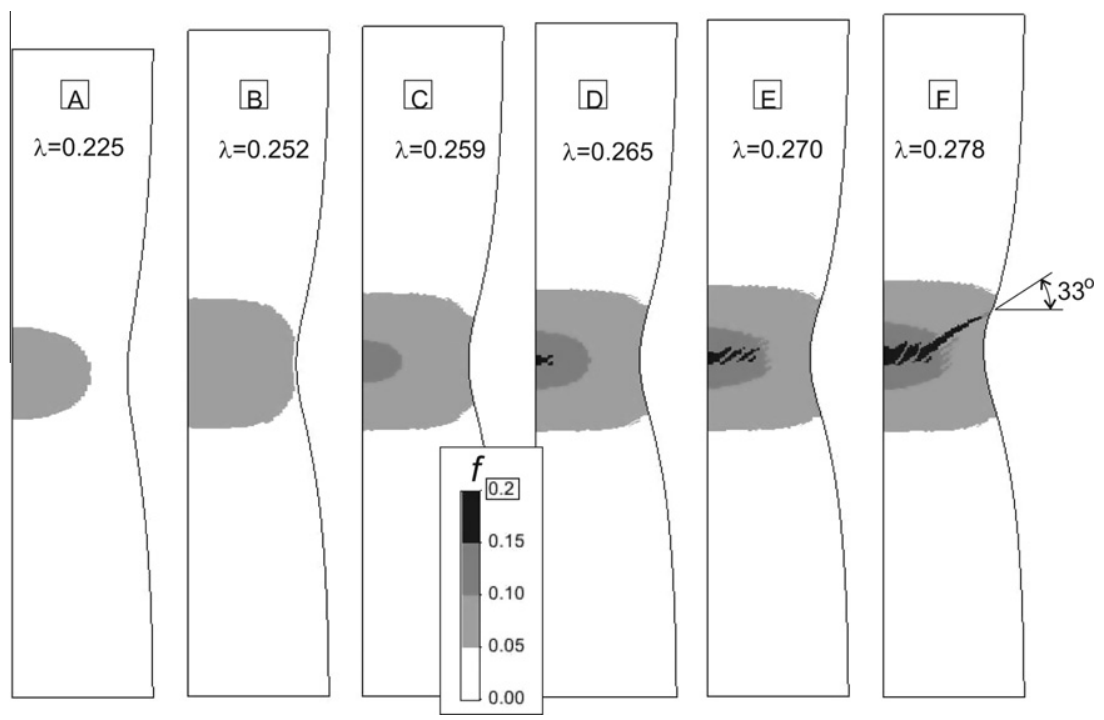

Fig. 10. Axisymmetric tension with $f_{N}=0.04 ., f^{*}$ as defined in Eq. (44) and Mesh 1 : void fraction distribution during the loading process, stages A, B, C, D, E and F correspond to the points displayed in Fig. 9 (b), $\lambda$ is the logarithm of the average bar stretching $(\lambda=\log [1+\Delta L / L])$. 
a larger value of $f_{N}$ gives earlier failure, the main features of the cup-cone failure mode are independent of the value specified for the volume fraction of void nucleating particles, at least in the circumstances analyzed here.

The critical angles between the localization band and the horizontal direction, in the central point of the bar during the onset of the first localization band, are $\pm 29^{\circ}$. The magnitude of these angles is greater than those for the material having a lower value of $f_{N}$. However, the final failure mode has an angle of approximately $33^{\circ}$, Fig. 10 , which is close to that observed in Fig. 8(a), stage E, calculated for a material having a lower value of $f_{N}$.

\subsection{Plane strain notched bar}

Calculations are carried out for the plane strain tensile response of a notched rectangular bar similar to those reported in Needleman and Tvergaard (1984) for a D-notched bar. The specimen geometry is shown in Fig. 11(a). One half of the bar is modeled. The numerical solutions are obtained using two quadrilateral finite element meshes: Mesh 1 and 2, as shown in Fig. 11(b) and (c), having a total of 4816 and 1011 finite elements respectively. These are structured meshes in the zone of height $L / 5$ close to the notch.

The material parameters are similar to those in Needleman and Tvergaard (1984) and they are: $\sigma_{y} / E=0.0033$, Poisson's ratio $v=0.3$, hardening exponent $N=0.1$, and $q_{1}=1.5$ and $q_{2}=1.0$. Also, in these calculations we take $f^{*} \equiv f$.

Void nucleation is taken to be strain controlled as described by Eqs. (16) and (17), with $f_{N}=0.04, \epsilon_{N}=0.3$ and $s_{N}=0.1$. The initial void volume fraction distribution is taken to be $f_{0}=0.0001$.

Fig. 12(a) compares load versus displacement curves obtained with the two meshes, both with $D_{0} / L=0.0015\left(D_{0}\right.$ is normalized by the half length of the specimen). For comparison purposes a solution obtained with Mesh 1 but without any weak discontinuity mode is also shown in Fig. 12(a). The solutions with the two finite element meshes including the weak discontinuity mode (having the same value of $D_{0}$ ), compare well over the entire range computed, illustrating the regularization effect obtained by including the enhanced mode. Without any enhanced mode, the development of failure is delayed and the results are strongly influenced by finite element mesh parameters (with quadrilateral finite element meshes), such as the size and aspect ratio of the elements.

Fig. 12(b) shows the fracture mode captured by Mesh 1 including the weak discontinuity modes. A localization instability first occurs at the notch root and a shear band initiates there. The failure mode consists of a shear band, starting at the notch root, which then propagates at an angle of approximately $125^{\circ}$. This shear band arrests and a shear band develops, at $40^{\circ}$ to $45^{\circ}$ with the horizontal direction, that intersects the center of the specimen.

The more dissipative response of the solution without discontinuity modes can be understood better by analyzing the development of the failure mode as shown in Fig. 13. Figs. 13(a) and (b) show results from calculations carried out for half of the specimen, the shaded region in Fig. 11(a), while for the calculation in Fig. 13(c) symmetry about the notch mid-plane was also imposed. Fig. 13(a) shows one configuration of the full specimen width consistent with the imposed boundary conditions. Another possible configuration of the full specimen, based on the same half-specimen calculation, has the shear bands emanating from both notches in the upper half of the specimen. The loss of symmetry between the two shear bands at each notch is due to the unsymmetric perturbation we introduced to force the development of one of the two possible shear band modes at the notch root $\left( \pm 125^{\circ}\right)$. When discontinuity modes are not included, the initial band at $125^{\circ}$ is not well developed, Fig. 13(b). A horizontal band then develops close to the notch surface. This gives a delay in shear band formation and consequently an increase in energy dissipation.

Fig. 13(c) shows a calculation in which shear band formation at the notch root was prescribed to occur at $-125^{\circ}$ and the calculation was carried out for one quarter of the specimen. Comparing the overall load versus engineering strain curves in Fig. 12(b) shows that the failure strain and overall dissipation are nearly the same for the two failure modes shown in Figs. 13(a) and (c). This indicates that the increased dissipation seen for the symmetric failure mode obtained when no discontinuity modes were included is not a consequence of the failure mode symmetry.
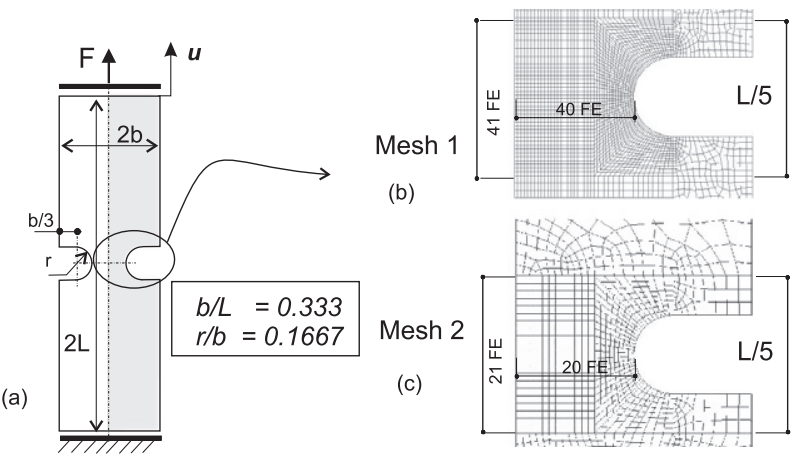

Fig. 11. (a) Sketch illustrating the boundary problem for the plane strain D-notched specimen. (b) The finer finite element Mesh 1 in the notch vicinity. (c) The coarser finite element Mesh 2 in the notch vicinity. 

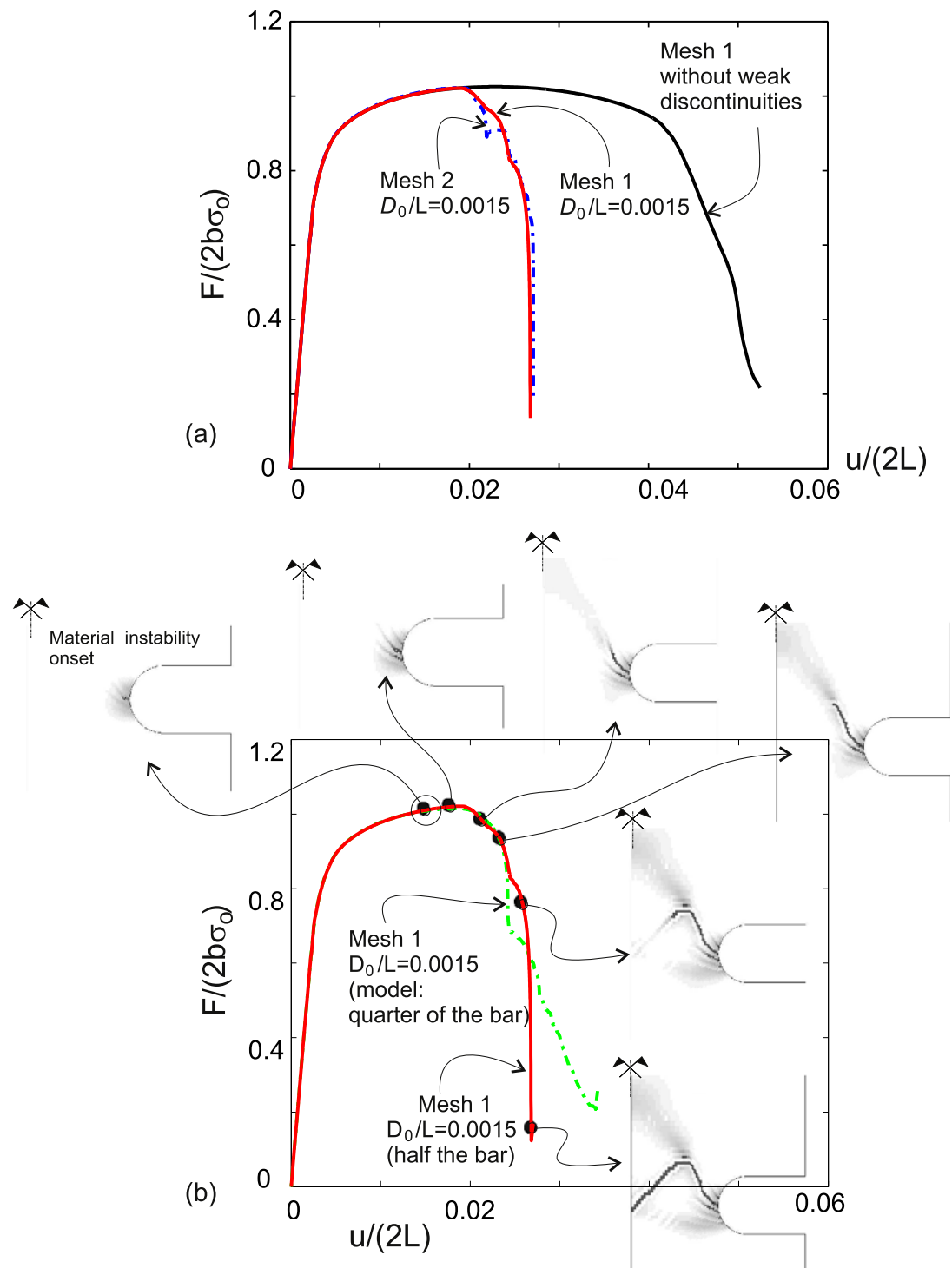

Fig. 12. Plane strain D-notched specimen. (a) Comparison of the specimen load versus strain curves for the meshes in Figs. 11(b) and (c). Also, shown is the response predicted using Mesh 1 without use of weak discontinuity modes. (b) Specimen load versus strain curves. The responses obtained by analyzing one half and one quarter of the specimen are compared. Contours of void volume fraction showing the evolution of the failure mode for the calculation of one half of the specimen using Mesh 1 with the discontinuity modes are also shown.

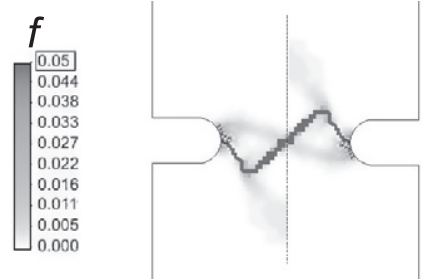

(a) Mesh 2

weak discontinuity solution with $\mathrm{D}_{0} / \mathrm{L}=0.0015$ (half-bar)

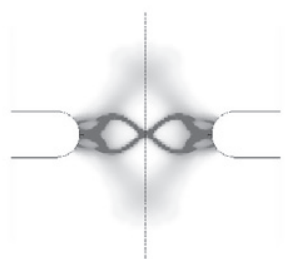

(b) Mesh 1 without weak discontinuity modes (half-bar)

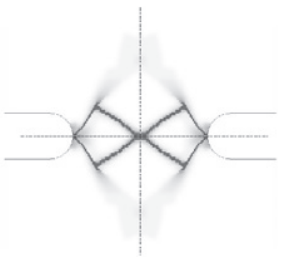

(c) Mesh 1 weak discontinuity solution with $\mathrm{D}_{0} / \mathrm{L}=0.0015$ (quarter-bar)

Fig. 13. Plane strain D-notched specimen. (a) The void volume fraction distribution obtained using Mesh 2 in Fig. 11(c) and modeling one half of the specimen. (b) The void volume fraction distribution obtained using Mesh 1 in Fig. 11(b) and modeling one half of the specimen. No weak discontinuity modes included. (c) The void volume fraction distribution using Mesh 1 in Fig. 11(b) and modeling one quarter of the specimen. 


\section{Concluding remarks}

We have presented a finite deformation generalization of the finite band formulation of Huespe et al. (2009). This method provides a unified framework for modeling the transition from a weak, localization discontinuity to a strong, fracture discontinuity with the accompanying loss of material stress carrying capacity. The same constitutive relation is used throughout the analysis with a specified localization band thickness serving as a characteristic length. The computed responses were rather independent of the finite element mesh design, including the orientation and the aspect ratio of the quadrilateral elements. Examples were presented using both structured and unstructured meshes. The numerical examples have shown that rather complex failure modes such as a cup-cone fracture can be represented.

Typically in structural applications, as in the examples here, the band width will be smaller than the element mesh size. In principle, however, the mesh size could be reduced so that it was smaller than the band width. In this case, consistent with the localization bifurcation analysis, the deformation state in the band needs to be taken to be constant through the band thickness. With this constraint, convergence could be obtained with increasing mesh refinement.

Key issues for further development are the extension to three dimensions and the automatic selection of the critical band orientation. It is worth emphasizing that, as in the small deformation case, the methodology here can be used with any rate independent constitutive relation that involves a localization instability possibly leading to a complete loss of stress carrying capacity.

\section{Acknowledgments}

A.E.H. and P.J.S. are grateful for financial support from ANPCyT and CONICET of Argentina through grants: PICT 200601232, PICT 2008-1228 and PIP 112-200901-00341. J.O. is grateful for financial support from the Spanish Ministry of Science and Innovation and the Catalan Government Research Department, under grants BIA2008-00411 and 2009 SGR 1510, respectively.

\section{References}

Alfaiate, J., Simone, A., Sluys, L.J., 2003. Non-homogeneous displacement jumps in strong embedded discontinuities. Int. J. Solids Struct. 40, $5799-5817$.

Armero, F., Garikipati, K., 1996. An analysis of strong discontinuities in multiplicative finite strain plasticity and their relation with the numerical simulation of strain localization. Int. J. Solids Struct. 33, 2863-2885.

Bargellini, R., Besson, J., Lorentz, E., Michel-Ponnelle, S., 2009. A non-local finite element based on volumetric strain gradient: application to ductile fracture. Comput. Mater. Sci. 45, 762-767.

Becker, R., Needleman, A., 1986. Effect of yield surface curvature on necking and failure in porous plastic solids. J. Appl. Mech. 53, $491-499$.

Belytschko, T., Moes, N., Usui, S., Parimi, C., 2001. Arbitrary discontinuities in finite elements. Int. J. Numer. Meth. Eng. $50,993-1013$.

Belytschko, T., Gracie, R., Ventura, G., 2009. A review of extended/generalized finite element methods for material modeling. Model. Simulat. Mater. Sci. Eng. 17. doi:10.1088/0965-0393/17/4/043001.

Benzerga, A.A., Leblond, J.B., 2010. Ductile fracture by void growth to coalescence. Adv. Appl. Mech. 44, 169-305.

Besson, J., Steglich, D., Brocks, W., 2001. Modeling of crack growth in round bar and plane strain specimens. Int. J. Solids Struct. 38, $8258-8284$.

Besson, J., Steglich, D., Brocks, W., 2003. Modeling of plane strain ductile rupture. Int. J. Plasticity 19, 1517-1541.

Cazes, F., Simatos, A., Coret, M., Combescure, A., 2010. A cohesive zone model which is energetically equivalent to a gradient-enhanced coupled damageplasticity model. Eur. J. Mech. A/Solids 29, 976-989.

Chu, C., Needleman, A., 1980. Void nucleation effects in biaxially stretched sheets. J. Eng. Mater. Technol. 102, 249-256.

Dvorkin, E.N., Cuitino, A.M., Gioia, G., 1990. Finite elements with displacement embedded localization lines insensitive to mesh size and distortions. Int. J. Numer. Meth. Eng. 30, 541-564.

Fleck, N.A., Hutchinson, J.W., 1997. Strain gradient plasticity. Adv. Appl. Mech. 33, 295-361.

Gao, H., Huang, Y., Nix, W.D., Hutchinson, J.W., 1999. Mechanism-based strain gradient plasticity: I Theory. J. Mech. Phys. Solids 47, $1239-1263$.

Gao, X., Zhang, T., Zhou, J., Graham, S.M., Hayden, M., Roe, C., 2011. On stress-state dependent plasticity modeling: significance of the hydrostatic stress, the third invariant of stress deviator and the non-associated flow rule. Int. J. Plasticity 27, 217-231.

Gurson, A., 1975. Plastic flow and fracture behavior of ductile materials incorporating void nucleation, growth and interaction. Ph.D. Thesis, Brown University.

Hadamard, J., 1903. Leçons sur la propagation des ondes et les equations de l'hydrodynamique. Librairie Scientifique A. Hermann et Fils, Paris.

Hill, R., 1962. Accelerations waves in solids. J. Mech. Phys. Solids 10, 1-16.

Hill, R., 1978. Aspects of invariance in solid mechanics. Adv. Appl. Mech. 18, 1-75.

Huespe, A.E., Needleman, A., Oliver, J., Sánchez, P.J., 2009. A finite thickness band method for ductile fracture analysis. Int. J. Plasticity $25,2349-2365$.

Li, H., Fu, M.W., Lu, J., Yang, H., 2011. Ductile fracture: experiments and computations. Int. J. Plasticity 27, 147-180.

Lubarda, V.A., 2002. Elastoplasticity Theory. CRC Press.

Mathur, K.K., Needleman, A., Tvergaard, V., 1994. Ductile failure analyses on massively parallel computers. Comput. Meth. Appl. Mech. Eng. 119, 283-309.

Mediavilla, J., Peerlings, R.H.J., Geers, M.G.D., 2006. Discrete crack modelling of ductile fracture driven by non-local softening plasticity. Int. J. Numer. Meth. Eng. 66, 661-688.

Nashon, K., Hutchinson, J.W., 2008. Modification of the Gurson model for shear failure. Eur. J. Mech. Solids A27, 1-17.

Needleman, A., Tvergaard, V., 1984. An analysis of ductile rupture in notched bars. J. Mech. Phys. Solids 32, $461-490$.

Oliver, J., 1996. Modelling strong discontinuities in solid mechanics via strain softening constitutive equations Part 2. Numerical simulation. Int. J. Numer. Meth. Eng. 39, 3601-3623.

Oliver, J., Huespe, A.E., Pulido, M.D.G., Samaniego, E., 2003. On the Strong discontinuity approach in finite deformation setting. Int. J. Numer. Meth. Eng. 56, $1051-1082$.

Oliver, J., Huespe, A.E., Samaniego, E., Chaves, E., 2004. Continuum approach to the numerical simulation of material failure in concrete. Int. J. Numer. Anal. Meth. Geomech. 28, 609-632.

Oliver, J., Huespe, A.E., Cante, J.C., 2008. An implicit/explicit integration scheme to increase computability of non-linear material and contact/friction problems. Comp. Meth. Appl. Mech. Eng. 197, 1865-1889.

Oliver, J., Huespe, A.E., Cante, J.C., Diaz, G., 2010. On the numerical resolution of the discontinuous material bifurcation problem. Int. J. Num. Meth. Eng. 83, 786-804. 
Ortiz, M., Leroy, Y., Needleman, A., 1987. A finite element method for localized failure analysis. Comput. Meth. Appl. Mech. Eng. 61, $189-214$.

Pardoen, T., Scheyvaerts, F., Simar, A., Tekolu, C., Onck, P.R., 2010. Multiscale modeling of ductile failure in metallic alloys. Comptes Rendus Physique 11, 326-345.

Rice, J., 1976. The localization of plastic deformation. In: Koiter, W. (Ed.). Amsterdam, North-Holland, pp. $207-220$.

Sánchez, P.J., Huespe, A.E., Oliver, J., 2008. On some topics for the numerical simulation of ductile fracture. Int. J. Plasticity 24, $1008-1038$.

Simo, J.C., 1998. Topics on the numerical simulation of plasticity. In: Ciarlet, P.G., Lions, J.L. (Eds.), Handbook of Numerical Analysis, VI. Amsterdam, NorthHolland, pp. 183-499.

Tvergaard, V., 1981. Influence of voids on shear band instabilities under plane strain conditions. Int. J. Fract. 17, 389-407.

Tvergaard, V., 1982. On localization in ductile materials containing spherical voids. Int. J. Fract. 18, $237-252$.

Tvergaard, V., Needleman, A., 1984. Analysis of the cup-cone fracture in a round tensile bar. Acta Metall. 32, 157-169.

Tvergaard, V., 1990. Material failure by void growth to coalescence. Adv. Appl. Mech. 27, 83-151. 